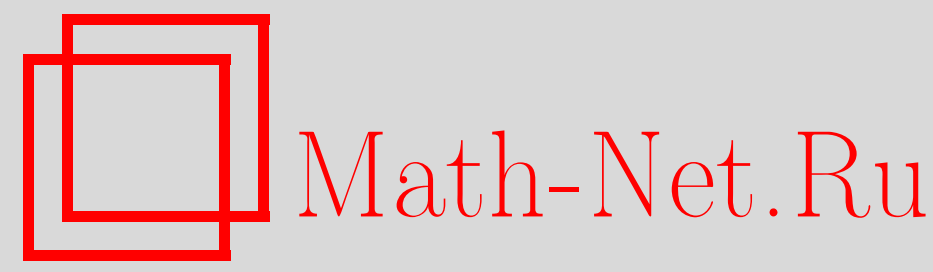

М. В. Милованов, Интегрируемость разрешимых алгебр Ли, Матем. сб., 1999, том 190, номер 5, 45-92

DOI: https://doi.org/10.4213/sm402

Использование Общероссийского математического портала Math-Net.Ru подразумевает, что вы прочитали и согласны с пользовательским соглашением

http: //www.mathnet.ru/rus/agreement

Параметры загрузки:

IP: 107.22 .136 .117

26 апреля 2023 г., 12:01:47 
УДК 512.813 .5

\author{
М.В. Милованов
}

\title{
Интегрируемость разрешимых алгебр Ли
}

\begin{abstract}
Для класса разрешимых алгебр Ли доказана справедливость известной гипотезы Мищенко-Фоменко об интегрируемости любой конечномерной алгебры Ли. Библиограбия: 15 названий.
\end{abstract}

\section{Введение}

Пусть $M$ - гладкое многообразие. Cимплектической структурой на $M$ называется замкнутая невырожденная дифференциальная 2-форма $\omega$. Пара $(M, \omega)$ называется симплектическим многообразием. Всякое симплектическое многообразие имеет четную размерность (см. [1]).

На симплектическом многообразии имеется естественный изоморфизм между векторными полями и 1-формами. Векторное поле $\operatorname{sgrad} f$, соответствуюшее дифференциалу функции $f \in C^{\infty}(M)$, назьвается гамильтоновым векторным полем. Система дифференциальных уравнений $\dot{x}=\operatorname{sgrad} f$ называется гамильтоновой системой, а функция $f$ - ее гамильтонианом.

Скобкой Пуассона функций $f, g \in C^{\infty}(M)$ называется функция $\{f, g\}$, определенная равенством $\{f, g\}=-\omega(\operatorname{sgrad} f, \operatorname{sgrad} g)$. Если $\{f, g\}=0$, то говорят, что функции $f, g$ находятся в инволющии. Функция $g$ является первым интеграломгамильтоновой системы $\dot{x}=\operatorname{sgrad} f$ тогда и только тогда, когда $\{f, g\}=0$. Если $g_{1}$ и $g_{2}$ - первые интегралы гамильтоновой системы, то и $\left\{g_{1}, g_{2}\right\}$ - первый интеграл этой системы.

Множество $C^{\infty}(M)$ образует бесконечномерную алгебру Ли относительно скобки Пуассона. Во многих конкретных ситуациях гамильтонова система обладает набором функционально независимых интегралов $g_{1}, \ldots, g_{m}$, линейная оболочка которых образует конечномерную алгебру Ли $\mathbf{G}$, т.е. $\left\{g_{i}, g_{j}\right\}=\sum_{k=1}^{m} C_{i j}^{k} g_{k}$, где $C_{i j}^{k}$ - константы. Особый интерес представляет случай, когда эта алгебра коммутативна и $m=\frac{1}{2} \operatorname{dim} M$. Мы попадаем при этом в сферу действия теоремы Лиувилля и будем говорить, что рассматриваемая гамильтонова система интедрируема в коммутативном смысле.

А. С. Мищенко и А.Т. Фоменко показали [2], что теорема Лиувилля допускает обобшение на случай некоммутативной алгебры $\mathbf{G}$ первых интегралов гамильтоновой системы, если условие $m=\frac{1}{2} \operatorname{dim} M$ заменить условием $\operatorname{dim} \mathbf{G}+\operatorname{ind} \mathbf{G}=$ $\operatorname{dim} M$, где целое число ind $\mathbf{G}$ называется индексом алгебры $\mathbf{G}$ и будет определено ниже. В этом случае говорят об интегрируемости гамильтоновой системь в некоммутативном смысле.

(C) М.В. МиловАнов 1999 
Пусть $\mathbf{G}$ - произвольная конечномерная алгебра Ли над полем действительных чисел, $\mathscr{G}$ - соответствуюшая ей связная группа Ли. Группа $\mathscr{G}$ действует сама на себе посредством сопряжения: $b \rightarrow a b a^{-1}$. Это действие индуцирует линейное действие $\mathscr{G}$ на ее алгебре $\mathbf{G}: \mathrm{Ad}: \mathscr{G} \rightarrow \mathrm{GL}(\mathbf{G})$, которое называется присоединенным представлением группы $\mathscr{G}$. Пусть $\mathbf{G}^{*}$ - коалгебра алгебры $\mathbf{G}$, т.е. пространство линейных функций $\xi: \mathbf{G} \rightarrow \mathbb{R}$ на $\mathbf{G}$. Определим коприсоединенное представление $\mathrm{Ad}^{*}: \mathscr{G} \rightarrow \mathrm{GL}\left(\mathbf{G}^{*}\right)$ следующим образом: $\left(\operatorname{Ad}_{g}^{*} \xi\right)(X)=\xi\left(\operatorname{Ad}_{g^{-1}} X\right), X \in \mathbf{G}$. Дифференциал коприсоединенного представления в единице $\mathbf{G}$ называется коприсоединенным представлением алгебры Ли $\mathbf{G}: \mathrm{ad}^{*}: \mathbf{G} \rightarrow \operatorname{Hom}\left(\mathbf{G}^{*}\right)$. При этом для $X, Y \in \mathbf{G}$ и $\xi \in \mathbf{G}^{*}$ имеет место равенство: $\left(\operatorname{ad}_{X}^{*} \xi\right)(Y)=-\xi([X, Y])$.

Для данного ковектора $\xi \in \mathbf{G}^{*}$ определим подпространство $\operatorname{Ann}(\xi)=\{X \in \mathbf{G}$ : $\left.\operatorname{ad}_{X}^{*} \xi=0\right\}$, которое называется аннулятором $\xi$. Число

$$
\text { ind } \mathbf{G}=\min _{\xi \in \mathbf{G}^{*}} \operatorname{dim} \operatorname{Ann}(\xi)
$$

называется индексом алгебры Ли $\mathbf{G}$.

На орбитах коприсоединенного представления имеется естественная симплектическая структура (форма Кириллова), в результате чего каждая орбита оказывается симплектическим многообразием с соответствуюшей скобкой Пуассона.

ОПРЕДЕЛЕНИЕ. Алгебра Ли $\mathbf{G}$ называется интегрируемой, если на $\mathbf{G}^{*}$ существует семейство функций $f_{1}, \ldots, f_{r}$ из $C^{\infty}\left(\mathbf{G}^{*}\right)$, удовлетворяющее следующим трем условиям:

1) $f_{1}, \ldots, f_{r}$ функционально независимы;

2) $r=\frac{1}{2}(\operatorname{dim} \mathbf{G}+\operatorname{ind} \mathbf{G})$;

3) $f_{1}, \ldots, f_{r}$ находятся попарно в инволюции на всех орбитах коприсоединенного представления.

Такое семейство будем назьвать полным инволютивным набором функиий на $\mathbf{G}^{*}$.

А.С. Мищенко и А.Т. Фоменко доказали [2], что если гамильтонова система интегрируема в некоммутативном смысле с помощюю интегрируемой алгебры Ли $\mathbf{G}$ своих интегралов, то эта система интегрируема и в коммутативном смысле. Ими же была высказана следующая общая гипотеза (см. [2]-[5]).

ГИПОТЕЗА. Любая конечномерная алгебра Ли интегрируема.

Доказательство этой гипотезы для того или иного класса алгебр Ли дает возможность не только редуцировать некоммутативную интегрируемость соответствующих гамильтоновых систем к коммутативной интегрируемости, но и позволяет предъявить большой запас интегрируемых гамильтоновых систем на орбитах коприсоединенного представления для таких алгебр. На этом пути появляются гамильтоновы системы, интересные с точки зрения приложений в геометрии, механике и физике.

Как известно, всякая алгебра Ли есть полупрямая сумма полупростой подалгебры и разрешимого идеала. Поэтому случаи, когда алгебра $\mathbf{G}$ полупроста или 
разрешима, можно считать основньми. Для любой полупростой алгебры Ли гипотезу доказали ее авторы. Но она до сих пор не доказана для произвольной разрешимой алгебры (детали см. в [4], [5]). Тем не менее, гипотеза доказана для всех нильпотентных алгебр [6] и многих бесконечных серий разрешимых ([7]-[10] и др.).

Главным результатом настоящей статьи является

ОСНОВНАЯ ТЕОРЕМА. Любая разрешимая алгебра Ли $\mathbf{G}$ интегрируема. При этом полный инволютивный набор на $\mathbf{G}^{*}$ можнн выцбрать из однородных функиий первой степени однородности, каждая из которых выражсается в явном виде в квадратурах.

Этот результат анонсирован автором в [11].

\section{§1. Индуктивный переход}

Пусть $\mathbf{G}$ - произвольная алгебра Ли над полем действительных чисел $\mathbb{R}$. Если $e_{1}, \ldots, e_{n}$ - базис $\mathbf{G}$, то через $e^{1}, \ldots, e^{n}$ обозначим соответствующий сопряженный базис в $\mathbf{G}^{*}$, так что $e^{j}\left(e_{i}\right)=\delta_{i j}$. Координаты ковектора $x \in \mathbf{G}^{*}$ в базисе $e^{1}, \ldots, e^{n}$ будем обозначать через $x_{1}, \ldots, x_{n}$.

Скобку Пуассона на орбитах коприсоединенного представления в $\mathbf{G}^{*}$ можно "склеить" в единую скобку Пуассона на всем пространстве $\mathbf{G}^{*}$ ( скобка Березина). Если $f, g \in C^{\infty}\left(\mathbf{G}^{*}\right)$, то положим по определению $\{f, g\}(x)=\left\{\left.f\right|_{\mathscr{O}(x)},\left.g\right|_{\mathscr{O}(x)}\right\}(x)$. При этом (см. [5; $\S 22])$, имеет место равенство

$$
\{f, g\}=C_{i j}^{k} x_{k} \frac{\partial g}{\partial x_{i}} \frac{\partial f}{\partial x_{j}}
$$

где $C_{i j}^{k}$ - структурные константы алгебры $\mathbf{G}$ в базисе $e_{1}, \ldots, e_{n}$.

Пусть $W$ - подпространство векторного пространства $V$, а $W^{*}$ и $V^{*}$ - соответствующие сопряженные пространства. Если $x \in V^{*}$, т.е. $x$ - линейная функция на $V$, то можно рассмотреть $\left.x\right|_{W}$ - ограничение $x$ на $W$. Ясно, что $\left.x\right|_{W}$ есть линейная функция на $W$, так что $\left.x\right|_{W} \in W^{*}$. В результате возникает естественное отображение $\pi: V^{*} \rightarrow W^{*}$ такое, что $\pi(x)=\left.x\right|_{W}, x \in V^{*}$. Легко видеть, что отображение $\pi$ - линейное.

Пусть теперь $f$-произвольная функция на $W^{*}$, т.е. $f: W^{*} \rightarrow \mathbb{R}$. Положим $\widetilde{f}=f \circ \pi$. Тогда $\widetilde{f}-$ вполне определенная функция на $V^{*}$, зависяшая лишь от выбора функции $f$.

Выберем в $W$ какой-нибудь базис $e_{1}, \ldots, e_{m}$ и дополним его произвольным образом до базиса $e_{1}, \ldots, e_{n}$ пространства $V$. Через $e_{W}^{1}, \ldots, e_{W}^{m}$ обозначим базис $W^{*}$, сопряженный к базису $e_{1}, \ldots, e_{m}$, а через $e_{V}^{1}, \ldots, e_{V}^{n}-$ базис $V^{*}$, сопряженньй к базису $e_{1}, \ldots, e_{n}$.

Пусть ковектор $x \in W^{*}$ имеет в базисе $e_{W}^{1}, \ldots, e_{W}^{m}$ координаты $x_{1}, \ldots, x_{m}$. Тогда $f=f(x)$ можно записать в виде $f=f\left(x_{1}, \ldots, x_{m}\right)$. Посмотрим, какой вид имеет функция $\widetilde{f}$ в базисе $e_{V}^{1}, \ldots, e_{V}^{n}$. Если ковектор $x \in V^{*}$ имеет в базисе 
$e_{V}^{1}, \ldots, e_{V}^{n}$ координаты $x_{1}, \ldots, x_{n}$, то $\widetilde{f}=\widetilde{f}\left(x_{1}, \ldots, x_{n}\right)$,

$$
\begin{aligned}
\widetilde{f}\left(x_{1}, \ldots, x_{n}\right) & =\widetilde{f}\left(x_{1} e_{V}^{1}+\cdots+x_{n} e_{V}^{n}\right) \\
& =f\left(\pi\left(x_{1} e_{V}^{1}+\cdots+x_{n} e_{V}^{n}\right)\right) \\
& =f\left(x_{1} \pi\left(e_{V}^{1}\right)+\cdots+x_{n} \pi\left(e_{V}^{n}\right)\right) \\
& =f\left(x_{1} e_{W}^{1}+\cdots+x_{m} e_{W}^{m}+x_{m+1} \cdot 0+\cdots+x_{n} \cdot 0\right) \\
& =f\left(x_{1} e_{W}^{1}+\cdots+x_{m} e_{W}^{m}\right)=f\left(x_{1}, \ldots, x_{m}\right) .
\end{aligned}
$$

Таким образом,

$$
\widetilde{f}\left(x_{1}, \ldots, x_{n}\right)=f\left(x_{1}, \ldots, x_{m}\right) .
$$

Равенство (1.2) означает, что функция $\widetilde{f}=\widetilde{f}\left(x_{1}, \ldots, x_{n}\right)$ получается из функции $f=f\left(x_{1}, \ldots, x_{m}\right)$, если последнюю рассматривать как функцию от переменных $x_{1}, \ldots, x_{n}$. Ясно, что $\frac{\partial \widetilde{f}}{\partial x_{i}}=0$ при $i>m$.

Если отождествить $W^{*}$ с линейной оболочкой $L\left(e_{V}^{1}, \ldots, e_{V}^{m}\right)$, то $W^{*} \subset V^{*}$ и $\left.\widetilde{f}\right|_{W^{*}}=f$. Это дает основание назьвать функцию $\widetilde{f}$ продолжением функиии $f$ на $V^{*}$. Равенство (1.2) говорит о том, что продолжение $f$ на $V^{*}$ снова можно обозначить буквой $f$.

Пусть теперь $H$ - произвольная подалгебра алгебры $\mathbf{G}, V=\mathbf{G}, W=H$. Если $f$ - функция на $H^{*}$, то ее продолжение на $\mathbf{G}^{*}$ будем обозначать той же буквой $f$.

Следующая важная лемма принадлежит В.В. Трофимову [8].

ЛЕмма 1.1. Если гладкие функции $f$ и $g$ на $H^{*}$ находятся в инволюиии на всех орбитах коприсоединенного представления в $H^{*}$, то их продолжсения на $\mathbf{G}^{*}$ будут в инволюции на всех орбитах коприсоединенного представления ${ }_{\theta} \mathbf{G}^{*}$.

ДокАЗАТЕЛЬСтво. Воспользуемся формулой (1.1). Пусть $a, b, c$ пробегают значения $1, \ldots, n ; i, j, k$ - значения $1, \ldots, m ; \alpha, \beta, \gamma$-значения $m+1, \ldots, n$. Тогда

$$
\begin{aligned}
C_{a b}^{c} x_{c} \frac{\partial g}{\partial x_{a}} \frac{\partial f}{\partial x_{b}}= & C_{\alpha \beta}^{c} x_{c} \frac{\partial g}{\partial x_{\alpha}} \frac{\partial f}{\partial x_{\beta}}+C_{i \beta}^{c} x_{c} \frac{\partial g}{\partial x_{i}} \frac{\partial f}{\partial x_{\beta}} \\
& +C_{\alpha i}^{c} x_{c} \frac{\partial g}{\partial x_{\alpha}} \frac{\partial f}{\partial x_{i}}+C_{i j}^{c} x_{c} \frac{\partial g}{\partial x_{i}} \frac{\partial f}{\partial x_{j}} \\
= & C_{i j}^{c} x_{c} \frac{\partial g}{\partial x_{i}} \frac{\partial f}{\partial x_{j}}=C_{i j}^{k} x_{k} \frac{\partial g}{\partial x_{i}} \frac{\partial f}{\partial x_{j}}=0
\end{aligned}
$$

Последнее равенство следует из того, что $f$ и $g$ находятся в инволюции на всех орбитах в $H^{*}$. Надо учесть также, что $\frac{\partial g}{\partial x_{\alpha}}=\frac{\partial f}{\partial x_{\beta}}=0$ и что $C_{i j}^{\gamma}=0$ в силу условия $\left[e_{i}, e_{j}\right] \in H$. Лемма доказана.

Пусть $\mathscr{O}$ - орбита коприсоединенного представления, проходящая через ковектор $\xi \in \mathbf{G}^{*}$. Тогда касательная плоскость $T_{\xi} \mathscr{O}$ к орбите $\mathscr{O}$ состоит из ковекторов вида $\operatorname{ad}_{X}^{*} \xi$, где $X$ пробегает $\mathbf{G}($ см. $[5 ; \S 22])$. Это означает, что $\operatorname{dim} \mathscr{O}=\operatorname{dim} \mathbf{G}-$ $\operatorname{dim} \operatorname{Ann}(\xi)$. Если $\mathscr{O}$ - орбита обшего положения (т.е. наибольшей размерности), 
то $\operatorname{dim} \mathscr{O}=\operatorname{dim} \mathbf{G}-$ ind $\mathbf{G}$. Другими словами, ind $\mathbf{G}$ совпадает с коразмерностью орбиты общего положения коприсоединенного представления в $\mathbf{G}^{*}$. Это утверждение раскрывает геометрический смысл понятия индекса алгебры $\mathbf{G}$.

В дальнейшем полезно иметь в виду, что если $\mathscr{O}$ - орбита обшего положения в $\mathbf{G}^{*}$, то

$$
\frac{1}{2}(\operatorname{dim} \mathbf{G}+\operatorname{ind} \mathbf{G})=\operatorname{codim} \mathscr{O}+\frac{1}{2} \operatorname{dim} \mathscr{O}
$$

Действительно,

$$
\operatorname{codim} \mathscr{O}+\frac{1}{2} \operatorname{dim} \mathscr{O}=\operatorname{ind} \mathbf{G}+\frac{1}{2}(\operatorname{dim} \mathbf{G}-\operatorname{ind} \mathbf{G})=\frac{1}{2}(\operatorname{dim} \mathbf{G}+\operatorname{ind} \mathbf{G})
$$

Пусть теперь $\mathbf{G}$ - произвольная разрешимая алгебра Ли размерности $n$.

Если $n=1$, то $\mathbf{G}-$ коммутативная алгебра, $\operatorname{dim} \mathscr{O}=0, \operatorname{codim} \mathscr{O}=1$ и $r=$ $\operatorname{codim} \mathscr{O}+\frac{1}{2} \operatorname{dim} \mathscr{O}=1$. Это означает, что полньй инволютивный набор на $\mathbf{G}^{*}$ должен состоять из одной функции, например из координатной функции $x_{1}$ на $\mathbf{G}^{*}$. Очевидно, $f_{1}=x_{1}$ - однородная функция первой степени однородности.

Предположим, что основная теорема уже доказана для разрешимых алгебр размерности меньше $n$. Пусть $\mathbf{G}_{0}$ - один из $(n-1)$-мерных идеалов алгебры $\mathbf{G}$. Выберем в $\mathbf{G}_{0}$ какой-либо базис $e_{1}, \ldots, e_{n-1}$ и дополним его до базиса $e_{1}, \ldots, e_{n-1}, e_{n}$ алгебры $\mathbf{G}$. По предположению индукции на $\mathbf{G}_{0}^{*}$ существует полный инволютивный набор $f_{1}, \ldots, f_{r_{0}}$, состоящий из однородных функций первой степени однородности. Их продолжения на $\mathbf{G}^{*}$ согласно лемме 1.1 находятся попарно в инволюции на всех орбитах коприсоединенного представления в $\mathbf{G}^{*}$ и, очевидно, остаются однородными функциями первой степени однородности.

Пусть $C_{i j}^{k}$ - структурные константы алгебры $\mathbf{G}$ в базисе $e_{1}, \ldots, e_{n}$. И пусть ковектор $x \in \mathbf{G}^{*}$ имеет в базисе $\mathbf{G}^{*}$, дуальном к базису $e_{1}, \ldots, e_{n}$, координаты $x_{1}, \ldots, x_{n}$. Рассмотрим матрицу $A=\left\|C_{i j}^{k} x_{k}\right\|$. Если $\mathscr{O}(x)-$ орбита коприсоединенного представления, проходящая через $x$, то $\operatorname{dim} \mathscr{O}(x)=\operatorname{rang} A(\mathrm{~cm} .[8 ; \S 2])$.

Соответствующую матрицу для идеала $\mathbf{G}_{0}$ и его базиса $e_{1}, \ldots, e_{n-1}$ обозначим через $A_{0}$. Если в матрице $A$ зачеркнуть последнюю строку и последний столбец, то получится матрица $A_{0}$.

Если $\mathscr{O}$ и $\mathscr{O}_{0}$ - орбиты общего положения в $\mathbf{G}^{*}$ и $\mathbf{G}_{0}^{*}$ соответственно, то из теоремы о ранге матрицы следует, что $\operatorname{dim} \mathscr{O}_{0} \leqslant \operatorname{dim} \mathscr{O} \leqslant \operatorname{dim} \mathscr{O}_{0}+2$. А так как каждая орбита коприсоединенного представления четномерна, то либо $\operatorname{dim} \mathscr{O}=\operatorname{dim} \mathscr{O}_{0}+2$, либо $\operatorname{dim} \mathscr{O}=\operatorname{dim} \mathscr{O}_{0}$.

В первом случае полный инволютивный набор на $\mathbf{G}_{0}^{*}$ можно рассматривать как полный инволютивный набор на $\mathbf{G}^{*}$. Действительно,

$$
\begin{aligned}
r & =\operatorname{codim} \mathscr{O}+\frac{1}{2} \operatorname{dim} \mathscr{O}=(n-\operatorname{dim} \mathscr{O})+\frac{1}{2}\left(\operatorname{dim} \mathscr{O}_{0}+2\right) \\
& =\left[n-\left(\operatorname{dim} \mathscr{O}_{0}+2\right)\right]+\frac{1}{2} \operatorname{dim} \mathscr{O}_{0}+1=\left[(n-1)-\operatorname{dim} \mathscr{O}_{0}\right]+\frac{1}{2} \operatorname{dim} \mathscr{O}_{0} \\
& =\operatorname{codim} \mathscr{O}_{0}+\frac{1}{2} \operatorname{dim} \mathscr{O}_{0}=r_{0} .
\end{aligned}
$$


Во втором случае полный инволютивный набор на $\mathbf{G}^{*}$ должен состоять из $r_{0}+1$ функций. В самом деле,

$$
\begin{aligned}
r & =\operatorname{codim} \mathscr{O}+\frac{1}{2} \operatorname{dim} \mathscr{O}=(n-\operatorname{dim} \mathscr{O})+\frac{1}{2} \operatorname{dim} \mathscr{O} \\
& =\left(n-\operatorname{dim} \mathscr{O}_{0}\right)+\frac{1}{2} \operatorname{dim} \mathscr{O}_{0}=\left[(n-1)-\operatorname{dim} \mathscr{O}_{0}\right]+\frac{1}{2} \operatorname{dim} \mathscr{O}_{0}+1 \\
& =\operatorname{codim} \mathscr{O}_{0}+\frac{1}{2} \operatorname{dim} \mathscr{O}_{0}+1=r_{0}+1 .
\end{aligned}
$$

ОПРЕДЕЛЕНИЕ. Разрешимую алгебру Ли $\mathbf{G}$ будем называть несводимой, если при любом выборе ее идеала $\mathbf{G}_{0}$ коразмерности 1 размерности орбит общего положения в $\mathbf{G}^{*}$ и $\mathbf{G}_{0}^{*}$ совпадают.

Проведенные выше рассуждения показьвают, что в дальнейшем можно ограничиться рассмотрением несводимых разрешимых алгебр.

Если алгебра $\mathbf{G}$ несводима, то к полному инволютивному набору на $\mathbf{G}_{0}^{*}$ после его продолжения на $\mathbf{G}^{*}$ необходимо добавить лишш одну подходящую функцию на $\mathbf{G}^{*}$. Эту недостающую функцию мы будем выбирать из инвариантов коприсоединенного представления на $\mathbf{G}^{*}$, следуя идее работы [6]. Действительно, всякий инвариант по своему определению постоянен на любой орбите и, следовательно, находится в инволюции со всякой функцией на $\mathbf{G}^{*}$.

\section{§2. Инварианты коприсоединенного представления}

Пусть $\mathbf{G}$ - произвольная алгебра Ли.

ОПРЕДЕЛЕНИЕ. Функция $f \in C^{\infty}\left(\mathbf{G}^{*}\right)$ называется инвариантом на $\mathbf{G}^{*}$, если она постоянна на всех орбитах коприсоединенного представления.

Конечно, интерес представляют лишь инварианты, которые не являются константами.

Пусть $e_{1}, \ldots, e_{n}$ - базис алгебры $\mathbf{G}, C_{i j}^{k}$ - структурные константы в этом базисе. И пусть $x_{1}, \ldots, x_{n}$ - координаты ковектора $x \in \mathbf{G}^{*}$ в сопряженном базисе $e^{1}, \ldots, e^{n}$. Известно (см. [5; $\left.\left.§ 14\right]\right)$, что функция $f \in C^{\infty}\left(\mathbf{G}^{*}\right)$ является инвариантом тогда и только тогда, когда она удовлетворяет следуюшей системе линейных дифференциальных уравнений в частных производных первого порядка:

$$
C_{i j}^{k} x_{k} \frac{\partial f}{\partial x_{j}}=0, \quad i=1, \ldots, n .
$$

Ранг матрицы $\left\|C_{i j}^{k} x_{k}\right\|$ этой системы совпадает с размерностью орбиты коприсоединенного представления, проходящей через ковектор $x=\left(x_{1}, \ldots, x_{n}\right) \in \mathbf{G}^{*}$ (см. $\S 1)$.

Система (2.1) есть частный случай системы вида

$$
\left\{\begin{array}{l}
X_{1}(f) \equiv a_{11} p_{1}+\cdots+a_{1 n} p_{n}=0 \\
\cdots \ldots \ldots \ldots \ldots \ldots \ldots \ldots \ldots \cdots \cdots \\
X_{m}(f) \equiv a_{m 1} p_{1}+\cdots+a_{m n} p_{n}=0
\end{array}\right.
$$


где $p_{k}=\frac{\partial f}{\partial x_{k}}, a_{i k}=a_{i k}\left(x_{1}, \ldots, x_{n}\right)$. Все нужные нам результаты теории таких систем изложены в книге $[12 ; \S 1]$, терминологии и обозначений которой мы будем придерживаться.

Если $\varphi=\varphi\left(x_{1}, \ldots, x_{n}, p_{1}, \ldots, p_{n}\right)$ и $\psi=\psi\left(x_{1}, \ldots, x_{n}, p_{1}, \ldots, p_{n}\right)$ - любые гладкие функции переменных $x_{1}, \ldots, x_{n}$ и $p_{1}, \ldots, p_{n}$, то определим их скобку Пуассона следующим равенством:

$$
(\varphi, \psi)=\sum_{s=1}^{n}\left(\frac{\partial \varphi}{\partial p_{s}} \frac{\partial \psi}{\partial x_{s}}-\frac{\partial \varphi}{\partial x_{s}} \frac{\partial \psi}{\partial p_{s}}\right)
$$

Можно считать, не ограничивая общности, что уравнения системы $(2.2)$ линейно независимы, т.е. не сушествует множителей $\lambda_{k}=\lambda_{k}\left(x_{1}, \ldots, x_{n}\right)$, среди которых есть отличные от нуля, таких, что имеет место соотношение

$$
\sum_{k=1}^{m} \lambda_{k} X_{k}(f)=0
$$

тождественное относительно $x_{1}, \ldots, x_{n}$.

Система (2.2) называется полной, если всевозможные скобки Пуассона $\left(X_{i}(f), X_{j}(f)\right)$ есть линейные комбинации левых частей уравнений $(2.2)$ :

$$
\left(X_{i}(f), X_{j}(f)\right)=\sum_{k=1}^{m} \lambda_{i j}^{k} X_{k}(f)
$$

где $\lambda_{i j}^{k}=\lambda_{i j}^{k}\left(x_{1}, \ldots, x_{n}\right)$.

Если система (2.2) полная, то при некоторых естественных ограничениях на коэффициенты $a_{i j}$ ее обшее решение можно записать (локально) в виде

$$
f=\psi\left(\psi_{1}, \ldots, \psi_{n-m}\right)
$$

где $\psi$ - произвольная гладкая функция, а $\psi_{1}, \ldots, \psi_{n-m}-$ функционально независимые (локально определенные) функции.

Вернемся к системе (2.1). Если положить $\frac{\partial f}{\partial x_{j}}=p_{j}$, то она запишется в виде

$$
C_{i j}^{k} x_{k} p_{j}=0, \quad i=1, \ldots, n
$$

Обозначим через $X_{i}=X_{i}\left(x_{1}, \ldots, x_{n}, p_{1}, \ldots, p_{n}\right)$ левую часть $i$-го уравнения системы (2.4).

ТЕОРема 2.1. Система (2.4) является полной для любой алгебры Ли $\mathbf{G}$. При этом

$$
\left(X_{\alpha}, X_{\beta}\right)=\sum_{s=1}^{n} C_{\alpha \beta}^{s} X_{s}, \quad \alpha, \beta=1, \ldots, n
$$


ДокАЗАТЕЛЬСТво. Так как $X_{\alpha}=C_{\alpha j}^{k} x_{k} p_{j}, X_{\beta}=C_{\beta j}^{k} x_{k} p_{j}$, то

$$
\begin{aligned}
\left(X_{\alpha}, X_{\beta}\right) & =\sum_{s=1}^{n}\left[\left(C_{\alpha s}^{k} x_{k}\right)\left(C_{\beta j}^{s} p_{j}\right)-\left(C_{\alpha j}^{s} p_{j}\right)\left(C_{\beta s}^{k} x_{k}\right)\right] \\
& =\sum_{s=1}^{n}\left(C_{\alpha s}^{k} C_{\beta j}^{s}-C_{\alpha j}^{s} C_{\beta s}^{k}\right) x_{k} p_{j} \\
& =\sum_{s=1}^{n}\left(-C_{\beta j}^{s} C_{s \alpha}^{k}-C_{\alpha j}^{s} C_{\beta s}^{k}\right) x_{k} p_{j} \\
& =\sum_{s=1}^{n}\left(-C_{\beta j}^{s} C_{s \alpha}^{k}-C_{j \alpha}^{s} C_{s \beta}^{k}\right) x_{k} p_{j} .
\end{aligned}
$$

Из тождества Якоби для алгебры $\mathbf{G}$ вытекает следуюшее соотношение между структурными константами:

$$
\sum_{s=1}^{n}\left(C_{\beta j}^{s} C_{s \alpha}^{k}+C_{j \alpha}^{s} C_{s \beta}^{k}+C_{\alpha \beta}^{s} C_{s j}^{k}\right)=0,
$$

или

$$
\sum_{s=1}^{n}\left(-C_{\beta j}^{s} C_{s \alpha}^{k}-C_{j \alpha}^{s} C_{s \beta}^{k}\right)=\sum_{s=1}^{n} C_{\alpha \beta}^{s} C_{s j}^{k} .
$$

Поэтому

$$
\left(X_{\alpha}, X_{\beta}\right)=\sum_{s=1}^{n} C_{\alpha \beta}^{s} C_{s j}^{k} x_{k} p_{j}=\sum_{s=1}^{n} C_{\alpha \beta}^{s} X_{s} .
$$

Теорема доказана.

Лемма 2.1. Пусть $\mathbf{G}-n$-мерная несводимая разрешимая алгебра Ли, $\mathbf{G}_{0}$

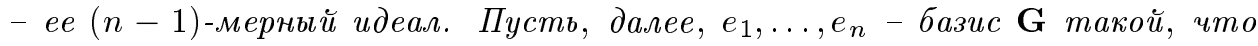
$e_{1}, \ldots, e_{n-1}-$ базис $\mathbf{G}_{0}$. И пусть $e^{1}, \ldots, e^{n}$ - соответствующий сопрязсенный базис коалгебры $\mathbf{G}^{*}$, в котором ковектор $x \in \mathbf{G}^{*}$ имеет координаты $x_{1}, \ldots, x_{n}$. Тогда на $\mathbf{G}^{*}$ существует (локально определенный) инвариант $f$, зависящий от $x_{n}$.

ДокАЗАТЕЛЬСТво. Если $\mathscr{O}$ и $\mathscr{O}_{0}$ - орбиты общего положения в $\mathbf{G}^{*}$ и $\mathbf{G}_{0}^{*}$ соответственно, то из несводимости $\mathbf{G}$ следует, что $\operatorname{dim} \mathscr{O}=\operatorname{dim} \mathscr{O}_{0}=m$. Поэтому в системе (2.4) можно оставить $m$ линейно независимых уравнений, причем полученная таким образом система остается полной и можно считать, что в ней отсутствует последнее уравнение из (2.4). Так как новая система имеет своими коэффициентами линейные функции, то ее общее решение имеет вид (2.3).

Покажем, что хотя бы один из инвариантов $\psi_{1}, \ldots, \psi_{n-m}$ зависит от $x_{n}$. Предположим противное. Тогда $\frac{\partial \psi_{1}}{\partial x_{n}}=\cdots=\frac{\partial \psi_{n-m}}{\partial x_{n}}=0$ и, следовательно, $\psi_{1}, \ldots, \psi_{n-m}$ есть решения системы для определения инвариантов на коалгебре $\mathbf{G}_{0}^{*}$. Но общее решение такой системы имеет вид

$$
f=h\left(\varphi_{1}, \ldots, \varphi_{n-m-1}\right)
$$


так что $\psi_{i}=h_{i}\left(\varphi_{1}, \ldots, \varphi_{n-m-1}\right), i=1, \ldots, n-m$. Это противоречит функциональной независимости $\psi_{1}, \ldots, \psi_{n-m}$. Лемма доказана.

Так как функции, входящие в полный инволютивньй набор на $\mathbf{G}_{0}^{*}$, после продолжения на $\mathbf{G}^{*}$ не зависят от $x_{n}$, то $f$ есть искомый инвариант на $\mathbf{G}^{*}$. Однако лемма 2.1 гарантирует лишш его локальное сушествование.

\section{§3. Алгебры индекса 1}

Пусть $\mathbf{G}$ - несводимая разрешимая алгебра Ли размерности $n, \mathbf{G}_{0}-$ ee $(n-1)$ мерньй идеал, $\mathscr{O}$ и $\mathscr{O}_{0}$ - орбиты общего положения в $\mathbf{G}^{*}$ и $\mathbf{G}_{0}^{*}$. Из несводимости алгебры $\mathbf{G}$ следует, что ее индекс ind $\mathbf{G}$ не может быть равен нулю. Действительно, в этом случае мы имели бы $\operatorname{dim} \mathscr{O}_{0}=\operatorname{dim} \mathscr{O}=n>\operatorname{dim} \mathbf{G}_{0}^{*}$, что невозможно. Поэтому простейшим является случай ind $\mathbf{G}=1$.

Итак, пусть ind $\mathbf{G}=1$, так что

$$
\operatorname{dim} \mathscr{O}=\operatorname{dim} \mathscr{O}_{0}=n-1 .
$$

Выберем в $\mathbf{G}_{0}$ какой-нибудь базис $e_{1}, \ldots, e_{n-1}$ и дополним его до базиса $e_{1}, \ldots, e_{n}$ алгебры $\mathbf{G}$. Так как коммутант алгебры $\mathbf{G}$ содержится в $\mathbf{G}_{0}$, то коэффициенты системы (2.4) для определения инвариантов на $\mathbf{G}^{*}$, соответствующей базису $e_{1}, \ldots, e_{n}$, могут зависеть от $x_{1}, \ldots, x_{n-1}$, но не зависят от $x_{n}$.

Если в матрице $\left\|C_{i j}^{k} x_{k}\right\|$ системы (2.4) зачеркнуть последнюю строку и последний столбец, то получится матрица системы для определения инвариантов на $\mathbf{G}_{0}^{*}$, соответствуюшей базису $e_{1}, \ldots, e_{n-1}$. Определитель этой матрицы с учетом (3.1) отличен от нуля. Выражая $p_{1}, \ldots, p_{n-1}$ через $p_{n}$ по правилу Крамера, получим

$$
p_{i}=a_{i} p_{n}, \quad i=1, \ldots, n-1
$$

где $a_{i}$ - однородные рациональные функции степени однородности 0 от переменных $x_{1} \ldots, x_{n-1}$. Ясно, что системы (2.4) и (3.2) эквивалентны.

Вернемся к системе (2.2). Она называется якобиевой, если для нее все скобки Пуассона $\left(X_{i}(f), X_{j}(f)\right)$ тождественно равны нулю. Ясно, что якобиевость является частным случаем понятия полноты. Из теории полных систем (см. [12; 1$]$ ) известно, что полнота системы (2.4) влечет за собой якобиевость системы (3.2). Поэтому из теоремы 2.1 следует, что система (3.2) якобиева.

Следующая лемма доказывается простым вычислением.

ЛЕмма 3.1. Система (3.2) является якобиевой тогда и только тогда, $\kappa о 2 \partial a$

$$
\frac{\partial a_{i}}{\partial x_{j}}=\frac{\partial a_{j}}{\partial x_{i}}, \quad i, j=1, \ldots, n-1 .
$$

TEOPEMA 3.1. Пусmb

$$
f=x_{1} a_{1}+\cdots+x_{n-1} a_{n-1}+x_{n} .
$$

Тогда $f$ есть решение системь (3.2). При этом $f$-однородная рачиональная функиия степени однородности 1, удовлетворяющая условию $\frac{\partial f}{\partial x_{n}}=1$. 
ДокАЗАТЕЛЬСТво. Так как $a_{i}$ - однородная функция нулевой степени однородности от переменных $x_{1}, \ldots, x_{n-1}$, то для нее справедлива формула Эйлера:

$$
x_{1} \frac{\partial a_{i}}{\partial x_{1}}+\cdots+x_{n-1} \frac{\partial a_{i}}{\partial x_{n-1}}=0, \quad i=1, \ldots, n-1 .
$$

Если $i=1, \ldots, n-1$, то

$$
\frac{\partial f}{\partial x_{i}}=a_{i}+\left(x_{1} \frac{\partial a_{1}}{\partial x_{i}}+\cdots+x_{n-1} \frac{\partial a_{n-1}}{\partial x_{i}}\right) .
$$

Поэтому из (3.3) следует, что

$$
\frac{\partial f}{\partial x_{i}}=a_{i}+\left(x_{1} \frac{\partial a_{i}}{\partial x_{1}}+\cdots+x_{n-1} \frac{\partial a_{i}}{\partial x_{n-1}}\right)
$$

откуда с учетом (3.5) получаем

$$
\frac{\partial f}{\partial x_{i}}=a_{i}, \quad i=1, \ldots, n-1
$$

Так как $a_{1}, \ldots, a_{n-1}$ не зависят от $x_{n}$, то $\frac{\partial f}{\partial x_{n}}=1$ и, следовательно, $f$ удовлетворяет системе (3.2). Однородность функции $f$ степени 1 есть очевидное следствие формулы (3.4). Теорема доказана.

Найденный инвариант $f$ функционально не зависит от функций, входящих в полньй инволютивный набор на $\mathbf{G}_{0}^{*}$, это и есть искомая недостающая функция на $\mathbf{G}^{*}$.

\section{§4. Алгебры произвольного индекса. Случай $\varphi_{1}=\cdots=\varphi_{q}=0$}

Изучение разрешимых алгебр Ли произвольного индекса опирается на специальный выбор базиса таких алгебр.

Будем обозначать через $L\left(e_{1}, \ldots, e_{k}\right)$ линейную оболочку векторов $e_{1}, \ldots, e_{k}$.

ЛЕмма 4.1. Для всякой разрешимой алгебрь Ли $\mathbf{G}$ существует базис $e_{1}, \ldots, e_{n}$ такой, что при любом $i=1, \ldots, n$ имеет место одна из следующих трех возможностей:

1) для кахсдого $X \in \mathbf{G}$

$$
\left[X, e_{i}\right]=\lambda e_{i}+\cdots
$$

где $\lambda=\lambda(X) \in \mathbb{R}$, а многоточие обозначает некоторую линейную комбинаиию $e_{1}, \ldots, e_{i-1} ;$ при этом $L\left(e_{1}, \ldots, e_{i-1}\right)$ является идеалом $\mathbf{G}$;

2) для кахдого $X \in \mathbf{G}$

$$
\begin{aligned}
{\left[X, e_{i-1}\right] } & =u e_{i-1}-v e_{i}+\cdots \\
{\left[X, e_{i}\right] } & =v e_{i-1}+u e_{i}+\cdots
\end{aligned}
$$


где $u=u(X) \in \mathbb{R}, v=v(X) \in \mathbb{R}$, а многоточия обозначают некоторые линейнье комбиначии $e_{1}, \ldots, e_{i-2} ;$ при этом $L\left(e_{1}, \ldots, e_{i-2}\right)$ является идеалом $\mathbf{G} u$ $v(X)=0$ не для всех $X \in \mathbf{G}$;

3) для кахдого $X \in \mathbf{G}$

$$
\begin{aligned}
{\left[X, e_{i}\right] } & =u e_{i}-v e_{i+1}+\cdots \\
{\left[X, e_{i+1}\right] } & =v e_{i}+u e_{i+1}+\cdots
\end{aligned}
$$

где $и=u(X) \in \mathbb{R}, v=v(X) \in \mathbb{R}$, а многоточия обозначают некоторые линейнье комбинации $e_{1}, \ldots, e_{i-1} ;$ при әтом $L\left(e_{1}, \ldots, e_{i-1}\right)$ является идеалом $\mathbf{G} u$ $v(X)=0$ не для всех $X \in \mathbf{G}$.

Если при этом алгебра $\mathbf{G}$ имеет коммутант размерности $s>0$, то $e_{1}, \ldots, e_{s}-$ базис коммутанта.

Описанньй в лемме 4.1 базис будем называть каноническим базисом алгебры G. Его сушествование без труда выводится из теоремы Ли о разрешимых алгебрах Ли, примененной к присоединенному представлению $X \rightarrow \operatorname{ad} X$ алгебры $\mathbf{G}$. Легко видеть, что канонический базис данной алгебры определен не однозначно.

Пусть $\mathbf{G}$ - произвольная несводимая разрешимая алгебра Ли, у которой ind $\mathbf{G}=$ $q+1>1$. Выберем и зафиксируем какой-либо канонический базис $e_{1}, \ldots, e_{n}$ алгебры $\mathbf{G}$ и рассмотрим соответствуюшую ему систему для определения инвариантов на $\mathbf{G}^{*}$. Если $A$ - матрица этой системы, то $\operatorname{rang} A=n-q-1$.

Обозначим через $v_{1}, \ldots, v_{n}$ систему столбцов матрицы $A$. Выберем из них все столбцы, которые состоят из одних нулей или являются линейными комбинациями столбцов, им предшествуюших. Легко видеть, что количество выбранных столбцов равно $q+1$. Среди них (в силу несводимости $\mathbf{G}$ ) будет и столбец $v_{n}$. Пусть $v_{\alpha_{1}}, \ldots, v_{\alpha_{q}}, v_{n}, \alpha_{1}<\alpha_{2}<\cdots<\alpha_{q},-$ выбранные столбцы. Так как оставшиеся столбцы матрицы $A$ линейно независимы, то система для определения инвариантов позволяет выразить все $\frac{\partial f}{\partial x_{i}}, i \neq \alpha_{1}, \ldots, \alpha_{q}, n$, через $\frac{\partial f}{\partial x_{\alpha_{1}}}, \ldots, \frac{\partial f}{\partial x_{\alpha_{q}}}, \frac{\partial f}{\partial x_{n}}$.

Пусть $\operatorname{dim}[\mathbf{G}, \mathbf{G}]=s$. Так как случай $s=0$ интереса не представляет, будем считать, что $s>0$. Если положить $\frac{\partial f}{\partial x_{i}}=p_{i}, i=1, \ldots, n$, то

$$
p_{i}=a_{i \alpha_{1}} p_{\alpha_{1}}+\cdots+a_{i \alpha_{q}} p_{\alpha_{q}}+a_{i n} p_{n}, \quad i=1, \ldots, n-1, i \neq \alpha_{1}, \ldots, \alpha_{q}
$$

где $a_{i \alpha_{1}}, \ldots, a_{i \alpha_{q}}, a_{i n}$ - однородные рациональные функции степени однородности 0 от переменных $x_{1}, \ldots, x_{s}$. Нетрудно убедиться, что $a_{i \alpha_{1}}=0$ при $i>\alpha_{1}$, $a_{i \alpha_{2}}=0$ при $i>\alpha_{2}, \ldots, a_{i \alpha_{q}}=0$ при $i>\alpha_{q}$.

Следующая теорема играет ключевую роль во многих дальнейших рассуждениях.

ТЕОРема 4.1. Коэффициенты $a_{i \alpha_{j}}, i=1, \ldots, n-1, i \neq \alpha_{1}, \ldots, \alpha_{q}, j=$ $1, \ldots, q$, системы (4.1) имеют следующий вид:

$$
a_{i \alpha_{j}}=a_{i \alpha_{j}}^{\prime} x_{\alpha_{j}}+a_{i \alpha_{j}}^{\prime \prime},
$$

где функиии $a_{i \alpha_{j}}^{\prime}, a_{i \alpha_{j}}^{\prime \prime}$ не зависят от переменных $x_{\alpha_{j}}, x_{\alpha_{j}+1}, x_{\alpha_{j}+2}, \ldots, x_{n}$.

Теорема 4.1 будет доказана в следуюшем параграфе. 
СлЕДСТВИЕ 4.1. Коэффициенты $a_{i \alpha_{j}}, i=1, \ldots, n-1, i \neq \alpha_{1}, \ldots, \alpha_{q}, j=$ $1, \ldots, q$, системь (4.1) можно записать в виде

$$
a_{i \alpha_{j}}=a_{i \alpha_{j}}^{\prime} x_{\alpha_{j}}+a_{i \alpha_{j}}^{\prime \prime}
$$

әде функиии $a_{i \alpha_{j}}^{\prime}, a_{i \alpha_{j}}^{\prime \prime}$ не зависят от $x_{\alpha_{j}}, x_{\alpha_{j+1}}, \ldots, x_{\alpha_{q}}, x_{n}$.

С этого момента нам будет удобно перейти к новым обозначениям переменных $x_{1}, \ldots, x_{n}$ : переменные $x_{\alpha_{1}}, \ldots, x_{\alpha_{q}}, x_{n}$ будем обозначать через $y_{1}, \ldots, y_{q}, y_{q+1}$ соответственно. Все прочие переменные из $x_{1}, \ldots, x_{n}$ обозначим (в порядке возрастания номеров) через $x_{1}, \ldots, x_{m}$. Таким образом, $m+q+1=n$.

В новых обозначениях система (4.1) принимает вид

$$
\frac{\partial f}{\partial x_{i}}=a_{i 1} \frac{\partial f}{\partial y_{1}}+\cdots+a_{i q} \frac{\partial f}{\partial y_{q}}+a_{i q+1} \frac{\partial f}{\partial y_{q+1}}, \quad i=1, \ldots, m
$$

где $a_{i j}=a_{i j}\left(x_{1}, \ldots, x_{m}, y_{1}, \ldots, y_{q}\right)$ - однородные рациональные функции степени однородности 0 , а $f=f\left(x_{1}, \ldots, x_{m}, y_{1}, \ldots, y_{q}, y_{q+1}\right)$ - искомая функция.

Новую форму принимает и следствие 4.1 .

СлЕДСТВИЕ 4.1'. Коэффициенты $a_{i j}, i=1, \ldots, m, j=1, \ldots, q$, системь (4.2) мохсно записать в виде

$$
a_{i j}=a_{i j}^{\prime} y_{j}+a_{i j}^{\prime \prime}
$$

где функиии $a_{i j}^{\prime}, a_{i j}^{\prime \prime}$ не зависят от $y_{j}, y_{j+1}, \ldots, y_{q}, y_{q+1}$.

Из теоремы 2.1 вытекает якобиевость системы (4.2). Условия якобиевости систем такого вида приведены в $[13 ; \S 6]$.

ЛЕмма 4.2. Система (4.2) является якобиевой тогда и только тогда, $\kappa о г \partial а$

$$
\frac{\partial a_{i k}}{\partial x_{j}}-\frac{\partial a_{j k}}{\partial x_{i}}+\sum_{t=1}^{q}\left(a_{i t} \frac{\partial a_{j k}}{\partial y_{t}}-a_{j t} \frac{\partial a_{i k}}{\partial y_{t}}\right)=0, \quad i, j=1, \ldots, m, k=1, \ldots, q+1
$$

Учитьвая теорему 3.1, доказанную для алгебр индекса 1, будем искать решение $f$ системы (4.2) в виде однородной функции степени однородности 1 , удовлетворяющей дополнительному условию $\frac{\partial f}{\partial y_{q+1}}=1$.

Предположим, что такое решение существует. Так как $f$ - функция первой степени однородности, то

$$
f=\sum_{i=1}^{m} x_{i} \frac{\partial f}{\partial x_{i}}+\sum_{k=1}^{q+1} y_{k} \frac{\partial f}{\partial y_{k}}
$$

В то же время $f$ удовлетворяет системе (4.2) и потому

$$
\frac{\partial f}{\partial x_{i}}=\sum_{k=1}^{q+1} a_{i k} \frac{\partial f}{\partial y_{k}}, \quad i=1, \ldots, m
$$


Из (4.4) и (4.5) получаем:

$$
f=\sum_{i=1}^{m} x_{i}\left(\sum_{k=1}^{q+1} a_{i k} \frac{\partial f}{\partial y_{k}}\right)+\sum_{k=1}^{q+1} y_{k} \frac{\partial f}{\partial y_{k}}=\sum_{k=1}^{q+1}\left(\sum_{i=1}^{m} x_{i} a_{i k}+y_{k}\right) \frac{\partial f}{\partial y_{k}}
$$

Если положить

$$
\varphi_{k}=\sum_{i=1}^{m} x_{i} a_{i k}+y_{k}, \quad k=1, \ldots, q+1
$$

TO

$$
f=\sum_{k=1}^{q+1} \varphi_{k} \frac{\partial f}{\partial y_{k}}
$$

Так как по условию $\frac{\partial f}{\partial y_{q+1}}=1$, то

$$
f=\sum_{k=1}^{q+1} \varphi_{k} \frac{\partial f}{\partial y_{k}}+\varphi_{q+1}
$$

Мы видим, что если функция $f$ сушествует, то она удовлетворяет следуюшему линейному дифференциальному уравнению в частных производных первого порядка:

$$
\varphi_{1} \frac{\partial f}{\partial y_{1}}+\cdots+\varphi_{q} \frac{\partial f}{\partial y_{q}}=f-\varphi_{q+1}
$$

Обратно, пусть функция $f$ удовлетворяет уравнению (4.6), условию $\frac{\partial f}{\partial y_{q+1}}=1$ и является решением системы (4.2). Тогда $f$ удовлетворяет равенству (4.4) и, следовательно, есть однородная функция первой степени однородности.

ЛЕмма 4.3. Функиии $\varphi_{k}, k=1, \ldots, q+1$, удовлетворяют следующим соотношениям:

$$
\frac{\partial \varphi_{k}}{\partial x_{i}}=\sum_{t=1}^{q}\left(a_{i t} \frac{\partial \varphi_{k}}{\partial y_{t}}-\frac{\partial a_{i k}}{\partial y_{t}} \varphi_{t}\right)+\left(a_{i k}-\sum_{t=1}^{q} a_{i t} \frac{\partial y_{k}}{\partial y_{t}}\right), \quad i=1, \ldots, m
$$

$\Pi р и$ этом

$$
a_{i k}-\sum_{t=1}^{q} a_{i t} \frac{\partial y_{k}}{\partial y_{t}}= \begin{cases}0, & \text { ecлu } k=1, \ldots, q \\ a_{i k}, & \text { ecлu } k=q+1\end{cases}
$$


ДокАЗАТЕльство. Так как $a_{i k}$ - однородные функции степени однородности 0 , TO

$$
\sum_{j=1}^{m} x_{j} \frac{\partial a_{i k}}{\partial x_{j}}=-\sum_{j=1}^{q} y_{j} \frac{\partial a_{i k}}{\partial y_{j}}
$$

Рассмотрим $\frac{\partial \varphi_{k}}{\partial x_{i}}, k=1, \ldots, q+1, i=1, \ldots, m:$

$$
\begin{aligned}
\frac{\partial \varphi_{k}}{\partial x_{i}}= & \sum_{j=1}^{m} x_{j} \frac{\partial a_{j k}}{\partial x_{i}}+a_{i k}=\mid \text { см. }(4.3) \mid \\
= & \sum_{j=1}^{m} x_{j}\left(\frac{\partial a_{i k}}{\partial x_{j}}+\sum_{t=1}^{q}\left(a_{i t} \frac{\partial a_{j k}}{\partial y_{t}}-a_{j t} \frac{\partial a_{i k}}{\partial y_{t}}\right)\right)+a_{i k} \\
= & \sum_{j=1}^{m} x_{j} \frac{\partial a_{i k}}{\partial x_{j}}+\sum_{t=1}^{q}\left(a_{i t} \sum_{j=1}^{m} x_{j} \frac{\partial a_{j k}}{\partial y_{t}}-\frac{\partial a_{i k}}{\partial y_{t}} \sum_{j=1}^{m} x_{j} a_{j t}\right)+a_{i k}=\mid \mathrm{cM} . \\
= & -\sum_{j=1}^{q} y_{j} \frac{\partial a_{i k}}{\partial y_{j}}+\sum_{t=1}^{q}\left[a_{i t} \frac{\partial}{\partial y_{t}}\left(\sum_{j=1}^{m} x_{j} a_{j k}+y_{k}\right)\right. \\
& \left.-a_{i t} \frac{\partial y_{k}}{\partial y_{t}}-\frac{\partial a_{i k}}{\partial y_{t}}\left(\sum_{j=1}^{m} x_{j} a_{j t}+y_{t}\right)+y_{t} \frac{\partial a_{i k}}{\partial y_{t}}\right]+a_{i k} \\
= & \sum_{t=1}^{q}\left(a_{i t} \frac{\partial \varphi_{k}}{\partial y_{t}}-\frac{\partial a_{i k}}{\partial y_{t}} \varphi_{t}\right)+\left(a_{i k}-\sum_{t=1}^{q} a_{i t} \frac{\partial y_{k}}{\partial y_{t}}\right)
\end{aligned}
$$

Лемма доказана.

Вернемся к уравнению (4.6). Рассмотрим простейшпй случай, когда $\varphi_{1}=\cdots=$ $\varphi_{q}=0$. При таком предположении $f=\varphi_{q+1}$. Проверим, что функция $\varphi_{q+1}$ удовлетворяет системе (4.2).

При $k=q+1$ формула (4.7) принимает вид

$$
\frac{\partial \varphi_{q+1}}{\partial x_{i}}=\sum_{t=1}^{q}\left(a_{i t} \frac{\partial \varphi_{q+1}}{\partial y_{t}}-\frac{\partial a_{i q+1}}{\partial y_{t}} \varphi_{t}\right)+a_{i q+1}, \quad i=1, \ldots, m .
$$

А так как $\varphi_{1}=\cdots=\varphi_{q}=0$ и $\frac{\partial \varphi_{q+1}}{\partial y_{q+1}}=1$, то из (4.9) получаем

$$
\frac{\partial \varphi_{q+1}}{\partial x_{i}}=\sum_{t=1}^{q+1} a_{i t} \frac{\partial \varphi_{q+1}}{\partial y_{t}}, \quad i=1, \ldots, m
$$

т.е. $\varphi_{q+1}-$ решение системы $(4.2)$.

Доказана

ТЕОРема 4.2. Если $\varphi_{1}=\cdots=\varphi_{q}=0$, то функиия $f=\varphi_{q+1}$ есть решение системы (4.2). При этом $f$ - однородная рациональная функиия степени однородности 1 и $\frac{\partial f}{\partial y_{q+1}}=1$. 


\section{§5. Доказательство теоремы 4.1}

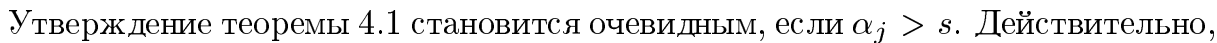
$a_{i \alpha_{j}}=a_{i \alpha_{j}}\left(x_{1}, \ldots, x_{s}\right)=a_{i \alpha_{j}}\left(x_{1}, \ldots, x_{\alpha_{j}-1}\right)$. Поэтому $a_{i \alpha_{j}}=a_{i \alpha_{j}}^{\prime} x_{\alpha_{j}}+a_{i \alpha_{j}}^{\prime \prime}$, где $a_{i \alpha_{j}}^{\prime}=0, a_{i \alpha_{j}}^{\prime \prime}=a_{i \alpha_{j}}$.

Будем считать для простоты обозначений, что $\alpha_{1}<\alpha_{2} \leqslant s, s<\alpha_{3}<\cdots<\alpha_{q}$. Тогда достаточно доказать утверждение теоремы только для коэффициентов $a_{i \alpha_{1}}$ и $a_{i \alpha_{2}}$. Мы ограничимся рассмотрением $a_{i \alpha_{1}}$, так как для $a_{i \alpha_{2}}$ все делается аналогично. При этом можно считать, что $\alpha_{1}>1$, так как в случае $\alpha_{1}=1$ все $a_{i \alpha_{1}}=0$ и $a_{i \alpha_{1}}=0 x_{\alpha_{1}}+0$.

Пусть $e_{1}, \ldots, e_{n}-$ выбранный канонический базис алгебры G. В соответствии с леммой 4.1 в случае $i=\alpha_{1}$ имеются три возможности, из которых первая наиболее простая для анализа. С нее и начнем.

Итак, пусть для каждого $X \in \mathbf{G}$

$$
\left[X, e_{\alpha_{1}}\right]=\lambda e_{\alpha_{1}}+\cdots,
$$

где $\lambda=\lambda(X) \in \mathbb{R}$, а многоточие обозначает некоторую линейную комбинацию $e_{1}, \ldots, e_{\alpha_{1}-1}$. При этом $L\left(e_{1}, \ldots, e_{\alpha_{1}-1}\right)$ является идеалом $\mathbf{G}$.

Рассмотрим вначале случай, когда $\lambda(X)=0$ для каждого $X \in \mathbf{G}$ (нулевой случай). Он заведомо имеет место, если $\mathbf{G}$ - нильпотентная алгебра. Покажем, что в нулевом случае коэффициенты $a_{i \alpha_{1}}$ есть функции от переменных $x_{1}, \ldots, x_{\alpha_{1}-1}$, так что $a_{i \alpha_{1}}=a_{i \alpha_{1}}^{\prime} x_{\alpha_{1}}+a_{i \alpha_{1}}^{\prime \prime}$, где $a_{i \alpha_{1}}^{\prime}=0, a_{i \alpha_{1}}^{\prime \prime}=a_{i \alpha_{1}}$.

Рассмотрим систему для определения инвариантов на $\mathbf{G}^{*}$ в каноническом базисе $e_{1}, \ldots, e_{n}$ :

$$
\sum_{j=1}^{n} C_{i j}^{k} x_{k} p_{j}=0, \quad i=1, \ldots, n .
$$

По условию она эквивалентна системе (4.1). Так как матрица $A$ системы (5.1) кососимметрична, то ее можно записать в виде

$$
A=\left[\begin{array}{cc}
A_{1} & A_{2} \\
-A_{2}^{T} & A_{3}
\end{array}\right]
$$

где $A_{1}$ - квадратная матрица порядка $\alpha_{1}$, а $T$ - значок транспонирования. Тогда коэффициенты матриц $A_{1}$ и $A_{2}$ (а потому и $-A_{2}^{T}$ ) - функции от $x_{1}, \ldots, x_{\alpha_{1}-1}$.

Так как в системе (4.1) коэффициенты $a_{i \alpha_{1}}=0$ при $i>\alpha_{1}$, то все $p_{i}$ при $i>\alpha_{1}$ выражаются через $p_{\alpha_{2}}, \ldots, p_{\alpha_{q}}, p_{n}$ :

$$
p_{i}=a_{i \alpha_{2}} p_{\alpha_{2}}+\cdots+a_{i \alpha_{q}} p_{\alpha_{q}}+a_{i n} p_{n}, \quad i=\alpha_{1}+1, \ldots, n-1, i \neq \alpha_{2}, \ldots, \alpha_{q}
$$

Если подставить в систему (5.1) вместо $p_{i}, i>\alpha_{1}$, их выражения через $p_{\alpha_{2}}, \ldots$, $p_{\alpha_{q}}, p_{n}$ по формулам (5.2), то получим новую систему, которая вместе с (5.2) эквивалентна (5.1). Эта новая система позволяет выразить $p_{1}, \ldots, p_{\alpha_{1}-1}$ через $p_{\alpha_{1}}, p_{\alpha_{2}}$, $\ldots, p_{\alpha_{q}}, p_{n}$. Если воспользоваться для этого правилом Крамера и тем, что коэффициенты матриц $A_{1},-A_{2}^{T}$ есть функции переменных $x_{1}, \ldots, x_{\alpha_{1}-1}$, то сразу видно, что

$$
p_{i}=a_{i \alpha_{1}} p_{\alpha_{1}}+\cdots+a_{i \alpha_{q}} p_{\alpha_{q}}+a_{i n} p_{n}, \quad i=1, \ldots, \alpha_{1}-1
$$


где $a_{i \alpha_{1}}=a_{i \alpha_{1}}\left(x_{1}, \ldots, x_{\alpha_{1}-1}\right)$. Этим завершается рассмотрение нулевого случая.

Предположим теперь, что $\lambda(X)=0$ не для всех $X \in \mathbf{G}$ (вещественный случай). Покажем, что это предположение приводит к противоречию, т.е. на самом деле вещественный случай не может иметь места.

По предположению

$$
\left[e_{i}, e_{\alpha_{1}}\right]=\lambda_{i} e_{\alpha_{1}}+\cdots, \quad i=1, \ldots, n
$$

где многоточие обозначает некоторую линейную комбинацию $e_{1}, \ldots, e_{\alpha_{1}-1}$. При этом $\lambda_{i}=0$ для всех $i \leqslant s$ (ввиду нильпотентности коммутанта разрешимой алгебры Ли), но для $i>s$ не все $\lambda_{i}=0$. Будем считать, не ограничивая общности, что $\lambda_{n} \neq 0$.

Если $\lambda_{i} \neq 0, s<i<n$, то положим

$$
e_{i}^{\prime}=-\frac{\lambda_{n}}{\lambda_{i}} e_{i}+e_{n}
$$

Тогда

$$
\left[e_{i}^{\prime}, e_{\alpha_{1}}\right]=0 e_{\alpha_{1}}+\cdots
$$

где многоточие есть линейная комбинация $e_{1}, \ldots, e_{\alpha_{1}-1}$. Если теперь в каноническом базисе $e_{1}, \ldots, e_{n}$ заменить каждый такой элемент $e_{i}$ на $e_{i}^{\prime}$, то получится новый канонический базис $\mathbf{G}$, удовлетворяющий условию

$$
\lambda_{1}=\cdots=\lambda_{n-1}=0, \quad \lambda_{n} \neq 0
$$

Обозначим его через $e_{1}^{\prime}, \ldots, e_{n}^{\prime}$.

При переходе от старого канонического базиса к новому матрица $A$ системы для определения инвариантов на $\mathbf{G}^{*}$ меняется, а вместе с ней меняется и матрица $A_{0}$, которая получается из $A$ после зачеркивания в ней последней строки и последнего столбца. Эти новые матрицы обозначим через $A^{\prime}$ и $A_{0}^{\prime}$. Очевидно, ранг матрицы $A$ остается при этом без изменения, т.е. $\operatorname{rang} A^{\prime}=n-q-1$. Так как алгебра $\mathbf{G}$ предполагается несводимой, то и $\operatorname{rang} A_{0}^{\prime}=n-q-1$. Поэтому последняя строка (столбец) матрицы $A^{\prime}$ есть линейная комбинация ее остальных строк (столбцов).

Вернемся к системе для определения инвариантов с матрицей $A$. Мы знаем (см. $\S 4)$, что столбец $A$ с номером $\alpha_{1}$ есть линейная комбинация предыдущих столбцов $A$. Следовательно, и столбец матрицы $A_{0}$ с номером $\alpha_{1}$ линейно выражается через предшествуюшие ему столбцы из $A_{0}$.

Покажем, что и в матрице $A_{0}^{\prime}$ столбец с номером $\alpha_{1}$ линейно выражается через предыдущие столбцы $A_{0}^{\prime}$. Так как матрица $A_{0}^{\prime}$ кососимметрична, то последнее утверждение равносильно аналогичному утверждению для строк из $A_{0}^{\prime}$.

Имея в виду, что $s$ - размерность коммутанта алгебры $\mathbf{G}$, обозначим через $A^{s}$ и $A_{0}^{s}$ матрицы, которые получаются из матриц $A$ и $A_{0}$ после зачеркивания их строк с номерами, большими $s$. Проследим, как изменится матрица $A_{0}^{s}$ при переходе от старого базиса к новому. У матрицы $A_{0}^{s}$ могут меняться лишш столбцы с номерами $s+1, \ldots, n-1$, причем меняются они в результате операций двух типов. Первая из них заключается в умножении одного из этих столбцов на ненулевое число $-\lambda_{n} / \lambda_{i}$. 
Операция второго типа прибавляет к результату умножения последний столбец матрицы $A^{s}$. Так как последний столбец $A^{s}$ есть линейная комбинация столбцов матрицы $A_{0}^{s}$, то мы имеем дело с элементарными преобразованиями столбцов матрицы $A_{0}^{s}$, которые, как известно, не меняют ранга матрицы. В качестве следствия получаем, что любая система строк матрицы $A_{0}^{s}$ не меняет своего ранга. Так как строка с номером $\alpha_{1}$ из $A_{0}$ (а потому и из $A_{0}^{s}$ ) линейно выражается через предыдущие, то из сказанного выше ясно, что строка с номером $\alpha_{1}$ матрицы $A_{0}^{\prime}$ линейно выражается через предыдущие строки $A_{0}^{\prime}$, что и требовалось установить.

Так как для нового базиса выполняется условие (5.3) и при этом $L\left(e_{1}^{\prime}, \ldots, e_{\alpha_{1}-1}^{\prime}\right)=L\left(e_{1}, \ldots, e_{\alpha_{1}-1}\right)-$ идеал алгебры $\mathbf{G}$, то элементы первых $\alpha_{1}$ строк матрицы $A_{0}^{\prime}$ есть функции от переменных $x_{1}^{\prime}, \ldots, x_{\alpha_{1}-1}^{\prime}$. Поэтому строка матрицы $A_{0}^{\prime}$ с номером $\alpha_{1}$ есть линейная комбинация первых $\alpha_{1}-1$ строк $A_{0}^{\prime}$ с коэффициентами в виде рациональных функций от $x_{1}^{\prime}, \ldots, x_{\alpha_{1}-1}^{\prime}$.

Рассмотрим теперь систему для определения инвариантов с матрицей $A^{\prime}$. Если из ее уравнения с номером $\alpha_{1}$ вычесть линейную комбинацию ее первых $\alpha_{1}-1$ уравнений с описанными выше коэффициентами, то получится уравнение вида

$$
\psi\left(x_{1}^{\prime}, \ldots, x_{\alpha_{1}}^{\prime}\right) \frac{\partial f}{\partial x_{n}^{\prime}}=0
$$

Так как первые $\alpha_{1}-1$ элементов последнего столбца матрицы $A^{\prime}$ есть функции от $x_{1}^{\prime}, \ldots, x_{\alpha_{1}-1}^{\prime}$, а его элемент с номером $\alpha_{1}$ зависит от $x_{\alpha_{1}}^{\prime}$ (ибо $\lambda_{n} \neq 0$ ), то

$$
\psi\left(x_{1}^{\prime}, \ldots, x_{\alpha_{1}}^{\prime}\right) \neq 0
$$

Поэтому (5.4) противоречит лемме 2.1. Это завершает изучение вешественного случая.

Пусть теперь для каждого $X \in \mathbf{G}$

$$
\begin{aligned}
{\left[X, e_{\alpha_{1}-1}\right] } & =u e_{\alpha_{1}-1}-v e_{\alpha_{1}}+\cdots \\
{\left[X, e_{\alpha_{1}}\right] } & =v e_{\alpha_{1}-1}+u e_{\alpha_{1}}+\cdots
\end{aligned}
$$

где $u=u(X) \in \mathbb{R}, v=v(X) \in \mathbb{R}$, а многоточия обозначают некоторые линейные комбинации $e_{1}, \ldots, e_{\alpha_{1}-2}$. При этом предполагается, что $L\left(e_{1}, \ldots, e_{\alpha_{1}-2}\right)$ - идеал алгебры $\mathbf{G}$ и $v(X)=0$ не для всех $X \in \mathbf{G}$ (комплексный случай). Тогда

$$
\begin{aligned}
{\left[e_{i}, e_{\alpha_{1}-1}\right] } & =u_{i} e_{\alpha_{1}-1}-v_{i} e_{\alpha_{1}}+\cdots \\
{\left[e_{i}, e_{\alpha_{1}}\right] } & =v_{i} e_{\alpha_{1}-1}+u_{i} e_{\alpha_{1}}+\cdots
\end{aligned}
$$

$i=1, \ldots, n$. Так как коммутант алгебры $\mathbf{G}$ нильпотентен, то $u_{i}=v_{i}=0$ при $i \leqslant s$. По условию при $i>s$ не все $v_{i}=0$. Поэтому будем считать, что $v_{n} \neq 0$, так как в противном случае вектор $e_{n}$ можно заменить на сумму $e_{n}$ и одного из векторов $e_{i}$, у которого $v_{i} \neq 0$.

Положим

$$
d_{i}= \begin{cases}e_{i}, & \text { если } v_{i}=0 \text { или } i=n ; \\ -\frac{v_{n}}{v_{i}} e_{i}+e_{n}, & \text { если } v_{i} \neq 0 \text { и } i<n .\end{cases}
$$


Тог да при $i<n$

$$
\begin{aligned}
{\left[d_{i}, d_{\alpha_{1}-1}\right] } & =u_{i}^{\prime} d_{\alpha_{1}-1}+\cdots, \\
{\left[d_{i}, d_{\alpha_{1}}\right] } & =u_{i}^{\prime} d_{\alpha_{1}}+\cdots,
\end{aligned}
$$

где $u_{i}^{\prime} \in \mathbb{R}$. Кроме того,

$$
\begin{aligned}
{\left[d_{n}, d_{\alpha_{1}-1}\right] } & =u_{n} d_{\alpha_{1}-1}-v_{n} d_{\alpha_{1}}+\cdots \\
{\left[d_{n}, d_{\alpha_{1}}\right] } & =v_{n} d_{\alpha_{1}-1}+u_{n} d_{\alpha_{1}}+\cdots
\end{aligned}
$$

Все многоточия обозначают линейные комбинации $d_{1}, \ldots, d_{\alpha_{1}-2}$. Ясно, что новый базис $d_{1}, \ldots, d_{n}$ остается каноническим базисом алгебры $\mathbf{G}$.

Если бы все $u_{i}^{\prime}=0, i=1, \ldots, n-1$, то возникла бы ситуация, аналогичная разобранной вьше для вешественного случая и приводящая к противоречию. Поэтому среди $u_{i}^{\prime}, i=s+1, \ldots, n-1$, не все равны нулю. Будем считать, что $u_{n-1}^{\prime} \neq 0$. В противном случае вектор $d_{n-1}$ можно заменить на сумму $d_{n-1}$ и одного из векторов $d_{i}$, у которого $u_{i}^{\prime} \neq 0$.

Положим

$$
e_{i}^{\prime}= \begin{cases}d_{i}, & \text { если } u_{i}^{\prime}=0 \text { или } i=n-1, n ; \\ -\frac{u_{n-1}^{\prime}}{u_{i}^{\prime}} d_{i}+d_{n-1}, & \text { если } u_{i}^{\prime} \neq 0 \text { и } i<n-1 .\end{cases}
$$

Тогда при $i<n-1$

$$
\begin{aligned}
{\left[e_{i}^{\prime}, e_{\alpha_{1}-1}^{\prime}\right] } & =0+\cdots, \\
{\left[e_{i}^{\prime}, e_{\alpha_{1}}^{\prime}\right] } & =0+\cdots .
\end{aligned}
$$

Кроме того,

$$
\begin{aligned}
{\left[e_{n-1}^{\prime}, e_{\alpha_{1}-1}^{\prime}\right] } & =u_{n-1}^{\prime} e_{\alpha_{1}-1}^{\prime}+\cdots \\
{\left[e_{n-1}^{\prime}, e_{\alpha_{1}}^{\prime}\right] } & =u_{n-1}^{\prime} e_{\alpha_{1}}^{\prime}+\cdots
\end{aligned}
$$

и

$$
\begin{array}{r}
{\left[e_{n}^{\prime}, e_{\alpha_{1}-1}^{\prime}\right]=u_{n} e_{\alpha_{1}-1}^{\prime}-v_{n} e_{\alpha_{1}}^{\prime}+\cdots} \\
{\left[e_{n}^{\prime}, e_{\alpha_{1}}^{\prime}\right]=v_{n} e_{\alpha_{1}-1}^{\prime}+u_{n} e_{\alpha_{1}}^{\prime}+\cdots}
\end{array}
$$

В формулах (5.5)-(5.7) все многоточия обозначают линейные комбинации $e_{1}^{\prime}, \ldots$, $e_{\alpha_{1}-2}^{\prime}$. При этом $L\left(e_{1}^{\prime}, \ldots, e_{\alpha_{1}-2}^{\prime}\right)$ является идеалом $\mathbf{G}$.

Новый базис $e_{1}^{\prime}, \ldots, e_{n}^{\prime}$ снова является каноническим базисом алгебры $\mathbf{G}$. Он лучше двух предыдуших базисов приспособлен к целям нашего исследования.

При переходе от старого базиса $e_{1}, \ldots, e_{n}$ к новому базису $e_{1}^{\prime}, \ldots, e_{n}^{\prime}$ матрица $A$ системы для определения инвариантов на $\mathbf{G}^{*}$ меняется, а вместе с ней меняется и матрица $A_{0}$, которая получается из $A$ после зачеркивания в ней последней строки и последнего столбца. Эти новые матрицы обозначим через $A^{\prime}$ и $A_{0}^{\prime}$. Ясно, что $\operatorname{rang} A^{\prime}=\operatorname{rang} A=n-q-1$. Так как алгебра $\mathbf{G}$ предполагается несводимой, то и $\operatorname{rang} A_{0}^{\prime}=n-q-1$. Поэтому последняя строка (столбец) матрицы $A^{\prime}$ есть линейная комбинация ее остальных строк (столбцов). Отсюда, в частности, следует, что в 
системе для определения инвариантов с матрицей $A^{\prime}$ можно зачеркнуть последнее уравнение.

Как и в вешественном случае, устанавливается, что столбец матрицы $A_{0}^{\prime}$ с номером $\alpha_{1}$ линейно выражается через предыдушие столбцы $A_{0}^{\prime}$. Совершенно аналогично получаем, что столбец $A_{0}^{\prime}$ с номером $\alpha_{2}$ есть линейная комбинация столбцов $A_{0}^{\prime}$ с номерами $1, \ldots, \alpha_{1}-1, \alpha_{1}+1, \ldots, \alpha_{2}-1$. Наконец, сходное рассуждение приводит к выводу, что в матрице $A_{0}^{\prime}$ столбцы с номерами $i=1, \ldots, s, i \neq \alpha_{1}, \alpha_{2}$, линейно независимы.

Рассмотрим систему для определения инвариантов на $\mathbf{G}^{*}$, отнесенную к базису $e_{1}^{\prime}, \ldots, e_{n}^{\prime}$. Она имеет матрицу $A^{\prime}$. Как мы уже знаем, последнее уравнение этой системы можно зачеркнуть. В результате получится однородная система, которая позволяет выразить все $p_{i}$ через $p_{\beta_{1}}, p_{\beta_{2}}, \ldots, p_{\beta_{q}}, p_{n}$, причем $\beta_{1}<\beta_{2}<\cdots<$ $\beta_{q}<n, \beta_{1}=\alpha_{1}, \beta_{2}=\alpha_{2}$ и $\beta_{3}>s$. Если воспользоваться для этого правилом Крамера, то сразу видно, что

$$
p_{i}=b_{i \beta_{1}} p_{\beta_{1}}+\cdots+b_{i \beta_{q}} p_{\beta_{q}}+b_{i n} p_{n}, \quad i=1, \ldots, n-1, i \neq \beta_{1}, \ldots, \beta_{q}
$$

где $b_{i \beta_{1}}, \ldots, b_{i \beta_{q}}, b_{i n}-$ функции переменных $x_{1}^{\prime}, \ldots, x_{s}^{\prime}$, причем $b_{i \beta_{1}}=0$ при $i>\beta_{1}=\alpha_{1}, b_{i \beta_{2}}=0$ при $i>\beta_{2}=\alpha_{2}$.

Покажем, что

$$
\begin{gathered}
b_{i \beta_{1}}=b_{i \alpha_{1}}=b_{i \alpha_{1}}^{\prime}\left(x_{1}^{\prime}, \ldots, x_{\alpha_{1}-1}^{\prime}\right) x_{\alpha_{1}}^{\prime}+b_{i \alpha_{1}}^{\prime \prime}\left(x_{1}^{\prime}, \ldots, x_{\alpha_{1}-1}^{\prime}\right), \\
i=1, \ldots, n-1, \quad i \neq \beta_{1}, \ldots, \beta_{q} .
\end{gathered}
$$

Пусть $A^{\prime}=\left(t_{i j}\right)$. Из равенств (5.5)-(5.7) следует, что

$$
\begin{aligned}
t_{i \alpha_{1}-1} & =0+\cdots, \quad i<n-1, & t_{i \alpha_{1}} & =0+\cdots, \quad i<n-1, \\
t_{n-1 \alpha_{1}-1} & =u_{n-1}^{\prime} x_{\alpha_{1}-1}^{\prime}+\cdots, & t_{n-1 \alpha_{1}} & =u_{n-1}^{\prime} x_{\alpha_{1}}^{\prime}+\cdots, \\
t_{n \alpha_{1}-1} & =u_{n} x_{\alpha_{1}-1}^{\prime}-v_{n} x_{\alpha_{1}}^{\prime}+\cdots, & t_{n \alpha_{1}} & =v_{n} x_{\alpha_{1}-1}^{\prime}+u_{n} x_{\alpha_{1}}^{\prime}+\cdots,
\end{aligned}
$$

где многоточия обозначают некоторые линейные функции от переменных $x_{1}^{\prime}, \ldots$, $x_{\alpha_{1}-2}^{\prime}$. А так как $L\left(e_{1}^{\prime}, \ldots, e_{\alpha_{1}-2}^{\prime}\right)$ - идеал $\mathbf{G}$, то при $j \leqslant \alpha_{1}-2$

$$
t_{i j}=t_{i j}\left(x_{1}^{\prime}, \ldots, x_{\alpha_{1}-2}^{\prime}\right)
$$

Для дальнейшего будет удобно представлять себе матрицу $A^{\prime}$ в виде

$$
A^{\prime}=\left[\begin{array}{cc}
A_{1} & -A_{2}^{T} \\
A_{2} & A_{3}
\end{array}\right],
$$

где $A_{1}$ - квадратная матрица порядка $\alpha_{1}$.

Так как система с матрицей $A^{\prime}$ эквивалентна системе $(5.8)$ и $b_{i \beta_{1}}=b_{i \alpha_{1}}=0$ при $i>\alpha_{1}$, то $p_{i}$ при $i>\alpha_{1}$ выражаются через $p_{\beta_{2}}, \ldots, p_{\beta_{q}}, p_{n}$ :

$$
p_{i}=b_{i \beta_{2}} p_{\beta_{2}}+\cdots+b_{i \beta_{q}} p_{\beta_{q}}+b_{i n} p_{n}, \quad i=\alpha_{1}+1, \ldots, n-1, i \neq \beta_{2}, \ldots, \beta_{q} .
$$


Если в систему с матрицей $A^{\prime}$ вместо $p_{i}, i=\alpha_{1}+1, \ldots, n-1, i \neq \beta_{2}, \ldots, \beta_{q}$, подставить соответствуюшие выражения из (5.11), то получится система, которая вместе с (5.11) эквивалентна системе с матрицей $A^{\prime}$. Эта новая система позволяет выразить $p_{1}, \ldots, p_{\beta_{1}-1}$ через $p_{\beta_{1}}, \ldots, p_{\beta_{q}}, p_{n}$. Мы можем зачеркнуть ее последнее уравнение (так как $\operatorname{rang} A^{\prime}=\operatorname{rang} A_{0}^{\prime}$ ); заметим, что в оставшейся системе коэффициенты при $p_{1}, \ldots, p_{\alpha_{1}}$ зависят лишь от переменных $x_{1}^{\prime}, \ldots, x_{\alpha_{1}-1}^{\prime}$. Единственное исключение составляет коэффициент $t_{n-1 \alpha_{1}}$ при $p_{\alpha_{1}}$, зависящий от $x_{\alpha_{1}}^{\prime}$ (см. (5.10)): $t_{n-1 \alpha_{1}}=u_{n-1}^{\prime} x_{\alpha_{1}}^{\prime}+\cdots$. Поэтому, выражая $p_{1}, \ldots, p_{\alpha_{1}-1}$ через $p_{\beta_{1}}, \ldots, p_{\beta_{q}}, p_{n}$ с помощью правила Крамера, получаем

$$
p_{i}=b_{i \beta_{1}} p_{\beta_{1}}+\cdots+b_{i \beta_{q}} p_{\beta_{q}}+b_{i n} p_{n}, \quad i=1, \ldots, \alpha_{1}-1,
$$

где

$$
b_{i \beta_{1}}=b_{i \alpha_{1}}=b_{i \alpha_{1}}^{\prime}\left(x_{1}^{\prime}, \ldots, x_{\alpha_{1}-1}^{\prime}\right) x_{\alpha_{1}}^{\prime}+b_{i \alpha_{1}}^{\prime \prime}\left(x_{1}^{\prime}, \ldots, x_{\alpha_{1}-1}^{\prime}\right), \quad i=1, \ldots, \alpha_{1}-1 .
$$

Так как $b_{i \beta_{1}}=b_{i \alpha_{1}}=0$ при $i>\alpha_{1}$, то тем самым формула (5.9) доказана.

Итак, утверждение теоремы 4.1 для коэффициентов $a_{i \alpha_{1}}$ пока что доказано нами не для исходного канонического базиса $e_{1}, \ldots, e_{n}$, а лишш для специально подобранного канонического базиса $e_{1}^{\prime}, \ldots, e_{n}^{\prime}$. В этой ситуации естественно воспользоваться формулами преобразования координат ковектора при изменении базиса алгебры $\mathbf{G}$ (см. [14; § 23]).

Пусть рассматриваются два базиса алгебры $\mathbf{G}-e_{1}, \ldots, e_{n}$ и $e_{1}^{\prime}, \ldots, e_{n}^{\prime}$. И пусть

$$
e_{j}=\sum_{i=1}^{n} c_{i j} e_{i}^{\prime}, \quad j=1, \ldots, n
$$

Обозначим через $e^{1}, \ldots, e^{n}$ и $e^{1^{\prime}}, \ldots, e^{n^{\prime}}$ соответствуюшие сопряженные базисы коалгебры $\mathbf{G}^{*}$. Если ковектор $x \in \mathbf{G}^{*}$ в базисе $e^{1}, \ldots, e^{n}$ имеет координаты $x_{1}, \ldots, x_{n}$, а в базисе $e^{1^{\prime}}, \ldots, e^{n^{\prime}}-$ координаты $x_{1}^{\prime}, \ldots, x_{n}^{\prime}$, то

$$
x_{j}=\sum_{i=1}^{n} c_{i j} x_{i}^{\prime}, \quad j=1, \ldots, n
$$

Таким образом, закон преобразования (5.13) имеет тот же вид (с теми же коэффициентами $c_{i j}$ ), что и закон преобразования (5.12).

В рассматриваемом нами случае

$$
e_{j}= \begin{cases}e_{j}^{\prime}, & \text { если } j=1, \ldots, s, \\ \sum_{i=s+1}^{n} c_{i j} e_{i}^{\prime}, & \text { если } j=s+1, \ldots, n .\end{cases}
$$

Поэтому

$$
x_{j}= \begin{cases}x_{j}^{\prime}, & \text { если } j=1, \ldots, s, \\ \sum_{i=s+1}^{n} c_{i j} x_{i}^{\prime}, & \text { если } j=s+1, \ldots, n .\end{cases}
$$


С помощью формул (5.14) новые координаты $x$ можно выразить через его старые координаты:

$$
x_{j}^{\prime}= \begin{cases}x_{j}, & \text { если } j=1, \ldots, s, \\ \sum_{i=s+1}^{n} d_{i j} x_{i}, & \text { если } j=s+1, \ldots, n .\end{cases}
$$

Рассмотрим произвольный инвариант $g=g\left(x_{1}^{\prime}, \ldots, x_{n}^{\prime}\right)$ на $\mathbf{G}^{*}$, записанный в новых координатах. По доказанному выше он должен удовлетворять системе (5.8) и, в частности, дифференциальному уравнению

$$
\frac{\partial g}{\partial x_{i}^{\prime}}=b_{i \beta_{1}} \frac{\partial g}{\partial x_{\beta_{1}}^{\prime}}+\cdots+b_{i \beta_{q}} \frac{\partial g}{\partial x_{\beta_{q}}^{\prime}}+b_{i n} \frac{\partial g}{\partial x_{n}^{\prime}}
$$

где $i=1, \ldots, s, i \neq \beta_{1}, \beta_{2}$. Если перейти к старым координатам, то наш инвариант запишется в виде функции $f=f\left(x_{1}, \ldots, x_{n}\right)$, которая будет удовлетворять уже другому дифференциальному уравнению, соответствуюшему уравнению (5.16). Выясним, что это за уравнение.

Нам понадобится следующий общий факт (см. [12; 1$]$ ). Пусть известно, что функция $g=g\left(x_{1}^{\prime}, \ldots, x_{n}^{\prime}\right)$ удовлетворяет уравнению

$$
b_{1} \frac{\partial g}{\partial x_{1}^{\prime}}+\cdots+b_{n} \frac{\partial g}{\partial x_{n}^{\prime}}=0
$$

где $b_{i}=b_{i}\left(x_{1}^{\prime}, \ldots, x_{n}^{\prime}\right)$. Введем новые независимые переменные

$$
x_{j}=\gamma_{j}\left(x_{1}^{\prime}, \ldots, x_{n}^{\prime}\right), \quad j=1, \ldots, n,
$$

причем

$$
x_{j}^{\prime}=h_{j}\left(x_{1}, \ldots, x_{n}\right), \quad j=1, \ldots, n .
$$

И пусть $f=g\left(h_{1}, \ldots, h_{n}\right)$, так что $f=f\left(x_{1}, \ldots, x_{n}\right)$. Тогда эта новая функция удовлетворяет следуюшему дифференциальному уравнению:

$$
\left.D\left(\gamma_{1}\right)\right|_{x_{j}^{\prime}=h_{j}} \frac{\partial f}{\partial x_{1}}+\cdots+\left.D\left(\gamma_{n}\right)\right|_{x_{j}^{\prime}=h_{j}} \frac{\partial f}{\partial x_{n}}=0
$$

где $D$ обозначает оператор $b_{1} \frac{\partial}{\partial x_{1}^{\prime}}+\cdots+b_{n} \frac{\partial}{\partial x_{n}^{\prime}}$.

В нашем случае

$$
\begin{aligned}
\gamma_{j}= \begin{cases}x_{j}^{\prime}, & \text { если } j=1, \ldots, s, \\
\sum_{i=s+1}^{n} c_{i j} x_{i}^{\prime}, & \text { если } j=s+1, \ldots, n,\end{cases} \\
h_{j}= \begin{cases}x_{j}, & \text { если } j=1, \ldots, s, \\
\sum_{i=s+1}^{n} d_{i j} x_{i}, & \text { если } j=s+1, \ldots, n .\end{cases}
\end{aligned}
$$


Возврашаемся к уравнению (5.16). Для него оператор $D$ таков:

$$
D=\frac{\partial}{\partial x_{i}^{\prime}}-b_{i \beta_{1}} \frac{\partial}{\partial x_{\beta_{1}}^{\prime}}-\ldots-b_{i \beta_{q}} \frac{\partial}{\partial x_{\beta_{q}}^{\prime}}-b_{i n} \frac{\partial}{\partial x_{n}^{\prime}}
$$

Поэтому при $j=1, \ldots, s$

$$
D\left(\gamma_{j}\right)=D\left(x_{j}^{\prime}\right)= \begin{cases}0, & \text { если } j \neq i, \beta_{1}, \beta_{2}, \\ 1, & \text { если } j=i, \\ -b_{i \beta_{1}}, & \text { если } j=\beta_{1}, \\ -b_{i \beta_{2}}, & \text { если } j=\beta_{2} .\end{cases}
$$

Учитывая, что $\beta_{1}=\alpha_{1}, \beta_{2}=\alpha_{2}$, получаем, что функция $f$ должна удовлетворять следуюшему дифференциальному уравнению:

$$
\begin{aligned}
\frac{\partial f}{\partial x_{i}}-\left.b_{i \alpha_{1}}\right|_{x_{j}^{\prime}=} & h_{j} \frac{\partial f}{\partial x_{\alpha_{1}}}-\left.b_{i \alpha_{2}}\right|_{x_{j}^{\prime}=h_{j}} \frac{\partial f}{\partial x_{\alpha_{2}}} \\
& -\left.D\left(\gamma_{s+1}\right)\right|_{x_{j}^{\prime}=h_{j}} \frac{\partial f}{\partial x_{s+1}}-\ldots-\left.D\left(\gamma_{n}\right)\right|_{x_{j}^{\prime}=h_{j}} \frac{\partial f}{\partial x_{n}}=0 .
\end{aligned}
$$

Так как $b_{i \alpha_{1}}, b_{i \alpha_{2}}, D\left(\gamma_{s+1}\right), \ldots, D\left(\gamma_{n}\right)$ есть функции от переменных $x_{1}^{\prime}, \ldots, x_{s}^{\prime}$ и $h_{j}=x_{j}$ при $j=1, \ldots, s$, то

$$
\begin{gathered}
\left.b_{i \alpha_{1}}\right|_{x_{j}^{\prime}=h_{j}}=b_{i \alpha_{1}}\left(x_{1}, \ldots, x_{s}\right),\left.\quad b_{i \alpha_{2}}\right|_{x_{j}^{\prime}=h_{j}}=b_{i \alpha_{2}}\left(x_{1}, \ldots, x_{s}\right) \\
\left.D\left(\gamma_{s+1}\right)\right|_{x_{j}^{\prime}=h_{j}}=D\left(\gamma_{s+1}\right)\left(x_{1}, \ldots, x_{s}\right), \ldots,\left.D\left(\gamma_{n}\right)\right|_{x_{j}^{\prime}=h_{j}}=D\left(\gamma_{n}\right)\left(x_{1}, \ldots, x_{s}\right)
\end{gathered}
$$

Поэтому уравнение (5.17) принимает вид:

$$
\begin{aligned}
\frac{\partial f}{\partial x_{i}}=b_{i \alpha_{1}}\left(x_{1}, \ldots, x_{s}\right) \frac{\partial f}{\partial x_{\alpha_{1}}}+b_{i \alpha_{2}}\left(x_{1}, \ldots, x_{s}\right) \frac{\partial f}{\partial x_{\alpha_{2}}} \\
\quad+D\left(\gamma_{s+1}\right)\left(x_{1}, \ldots, x_{s}\right) \frac{\partial f}{\partial x_{s+1}}+\cdots+D\left(\gamma_{n}\right)\left(x_{1}, \ldots, x_{s}\right) \frac{\partial f}{\partial x_{n}}
\end{aligned}
$$

Так как функция $f=f\left(x_{1}, \ldots, x_{n}\right)$ - инвариант, то она должна удовлетворять системе (4.1). Следовательно, при $i=1, \ldots, s, i \neq \alpha_{1}, \alpha_{2}$,

$$
\frac{\partial f}{\partial x_{i}}=a_{i \alpha_{1}} \frac{\partial f}{\partial x_{\alpha_{1}}}+a_{i \alpha_{2}} \frac{\partial f}{\partial x_{\alpha_{2}}}+\cdots+a_{i \alpha_{q}} \frac{\partial f}{\partial x_{\alpha_{q}}}+a_{i n} \frac{\partial f}{\partial x_{n}},
$$

а при $i>s$ все $\frac{\partial f}{\partial x_{i}}$ выражаются через $\frac{\partial f}{\partial x_{\alpha_{3}}}, \ldots, \frac{\partial f}{\partial x_{\alpha_{q}}}, \frac{\partial f}{\partial x_{n}}$.

Сравнивая уравнения (5.18) и (5.19), приходим к выводу, что

$$
a_{i \alpha_{1}}=b_{i \alpha_{1}}\left(x_{1}, \ldots, x_{s}\right)
$$


В противном случае $\frac{\partial f}{\partial x_{\alpha_{1}}}$ можно было бы линейно выразить через $\frac{\partial f}{\partial x_{\alpha_{2}}}, \ldots$, $\frac{\partial f}{\partial x_{\alpha_{q}}}, \frac{\partial f}{\partial x_{n}}$, а это противоречило бы тому, что $\mathbf{G}$ - алгебра индекса $q+1$.

Из (5.20) и (5.9) вытекает нужная формула:

$$
\begin{gathered}
a_{i \alpha_{1}}=a_{i \alpha_{1}}^{\prime}\left(x_{1}, \ldots, x_{\alpha_{1}-1}\right) x_{\alpha_{1}}+a_{i \alpha_{1}}^{\prime \prime}\left(x_{1}, \ldots, x_{\alpha_{1}-1}\right), \\
i=1, \ldots, n-1, \quad i \neq \beta_{1}, \ldots, \beta_{q} .
\end{gathered}
$$

Это завершает изучение комплексного случая.

В соответствии с леммой 4.1 для завершения доказательства теоремы 4.1 следовало бы разобрать третью возможность из этой леммы. Оказывается, однако, что новая ситуация ничем сушественньм не отличается от уже изученного комплексного случая.

§6. Алгебры произвольного индекса. Случай $\varphi_{1} \neq 0, \varphi_{2}=\cdots=\varphi_{q}=0$

Вернемся к системе (4.2) и уравнению (4.6). После уже рассмотренного случая $\varphi_{1}=\cdots=\varphi_{q}=0$ следующим по сложности является случай, когда одна из функций $\varphi_{1}, \ldots, \varphi_{q}$ отлична от нуля, а все остальные нулевые. Для простоты обозначений будем считать, что $\varphi_{1} \neq 0, \varphi_{2}=\cdots=\varphi_{q}=0$. Все прочие варианты рассматриваются аналогично.

При сделанном предположении уравнение (4.6) превращается в уравнение

$$
\varphi_{1} \frac{\partial f}{\partial y_{1}}=f-\varphi_{q+1},
$$

а формулы (4.7) принимают вид:

$$
\begin{gathered}
\frac{\partial \varphi_{1}}{\partial x_{i}}=\sum_{t=1}^{q} a_{i t} \frac{\partial \varphi_{1}}{\partial y_{t}}-\frac{\partial a_{i 1}}{\partial y_{1}} \varphi_{1}, \quad i=1, \ldots, m \\
0=-\frac{\partial a_{i k}}{\partial y_{1}} \varphi_{1}, \quad k=2, \ldots, q, \quad i=1, \ldots, m \\
\frac{\partial \varphi_{q+1}}{\partial x_{i}}=\sum_{t=1}^{q} a_{i t} \frac{\partial \varphi_{q+1}}{\partial y_{t}}-\frac{\partial a_{i q+1}}{\partial y_{1}} \varphi_{1}+a_{i q+1}, \quad i=1, \ldots, m .
\end{gathered}
$$

Так как $\varphi_{1} \neq 0$, то из $(6.3)$ следует, что

$$
\frac{\partial a_{i k}}{\partial y_{1}}=0, \quad k=2, \ldots, q, \quad i=1, \ldots, m
$$

Уравнение (6.1) можно рассматривать как обькновенное дифференциальное уравнение, считая все переменные, кроме $y_{1}$, параметрами. Хорошо известно, что уравнение

$$
\frac{d y}{d x}+P y=Q
$$


имеет общее решение вида

$$
y=e^{-\int P d x}\left(C+\int Q e^{\int P d x} d x\right),
$$

где $C$ - произвольная постоянная. В случае уравнения (6.1)

$$
y=f, x=y_{1}, P=-\frac{1}{\varphi_{1}}, Q=-\frac{\varphi_{q+1}}{\varphi_{1}}, C=C\left(x_{1}, \ldots, x_{m}, y_{2}, \ldots, y_{q+1}\right) .
$$

Поэтому обшее решение уравнения (6.1) таково:

$$
f=e^{\int \frac{1}{\varphi_{1}} d y_{1}}\left(C-\int \frac{\varphi_{q+1}}{\varphi_{1}} e^{-\int \frac{1}{\varphi_{1}} d y_{1}} d y_{1}\right) .
$$

Интегрируя по частям, имеем:

$$
-\int \frac{\varphi_{q+1}}{\varphi_{1}} e^{-\int \frac{1}{\varphi_{1}} d y_{1}} d y_{1}=\varphi_{q+1} e^{-\int \frac{1}{\varphi_{1}} d y_{1}}-\int \frac{\partial \varphi_{q+1}}{\partial y_{1}} e^{-\int \frac{1}{\varphi_{1}} d y_{1}} d y_{1} .
$$

Следовательно,

$$
f=\varphi_{q+1}+e^{\int \frac{1}{\varphi_{1}} d y_{1}}\left(C-\int \frac{\partial \varphi_{q+1}}{\partial y_{1}} e^{-\int \frac{1}{\varphi_{1}} d y_{1}} d y_{1}\right)
$$

Так как $\varphi_{1}$ и $\frac{\partial \varphi_{q+1}}{\partial y_{1}}$ не зависят от $y_{q+1}$, то можно считать, что каждый из интегралов, входящих в формулу (6.6), определен с точностью до произвольной функции от переменных $x_{1}, \ldots, x_{m}, y_{2}, \ldots, y_{q}$. Следующая теорема утверждает, что эти интегралы можно подобрать так, чтобы при $C=0$ формула (6.6) определяла функцию $f$, удовлетворяющую системе (4.2).

ТЕОремА 6.1. Если $\varphi_{1} \neq 0, \varphi_{2}=\cdots=\varphi_{q}=0$, то функиия

$$
f=\varphi_{q+1}-e^{\int \frac{1}{\varphi_{1}} d y_{1}} \int \frac{\partial \varphi_{q+1}}{\partial y_{1}} e^{-\int \frac{1}{\varphi_{1}} d y_{1}} d y_{1}
$$

при специальном выборе входящих в нее интегралов есть решение системъ (4.2). При этом $f$ - однородная функция степени однородности 1 и $\frac{\partial f}{\partial y_{q+1}}=1$. чтобы

ДокАЗАТЕльство. Так как $\frac{\partial f}{\partial y_{q+1}}=\frac{\partial \varphi_{q+1}}{\partial y_{q+1}}=1$, то необходимо добиться,

$$
\frac{\partial f}{\partial x_{i}}=\sum_{t=1}^{q} a_{i t} \frac{\partial f}{\partial y_{t}}+a_{i q+1}, \quad i=1, \ldots, m .
$$

Пусть $F_{1}^{0}$ - фиксированная гладкая функция, которая является одним из возможных значений интеграла $\int \frac{1}{\varphi_{1}} d y_{1}$. С помощью формул (6.2) и (6.5) доказыва-
ется, что

$$
\frac{\partial F_{1}^{0}}{\partial x_{i}}=\frac{a_{i 1}}{\varphi_{1}}+\sum_{t=2}^{q} a_{i t} \frac{\partial F_{1}^{0}}{\partial y_{t}}-c_{i}^{1}, \quad i=1, \ldots, m
$$

где $c_{i}^{1}$ - некоторая фуункция переменных $x_{1}, \ldots, x_{m}, y_{2}, \ldots, y_{q}$. 
Лемма 6.1. Существует функиия $\lambda_{1}=\lambda_{1}\left(x_{1}, \ldots, x_{m}, y_{2}, \ldots, y_{q}\right)$ такая, что функиия $F_{1}=F_{1}^{0}+\lambda_{1}$ удовлетворяет равенству

$$
\frac{\partial F_{1}}{\partial x_{i}}=\frac{a_{i 1}}{\varphi_{1}}+\sum_{t=2}^{q} a_{i t} \frac{\partial F_{1}}{\partial y_{t}}, \quad i=1, \ldots, m
$$

Функиия $\lambda_{1}$ находится в явном виде в квадратурах и зависит от выбора произвольной гладкой функиии от $q-1$ переменных.

Лемма 6.1 будет доказана в следуюшем параграфе.

В дальнейшем будем считать, что в формуле (6.7) $\int \frac{1}{\varphi_{1}} d y_{1}=F_{1}$.

Пусть $F_{2}^{0}$ - фиксированная гладкая функция, которая является одним из возможных значений интеграла $\int \frac{\partial \varphi_{q+1}}{\partial y_{1}} e^{-F_{1}} d y_{1}$. С помощью формул $(6.4),(6.5)$ и (6.10) доказывается, что

$\frac{\partial F_{2}^{0}}{\partial x_{i}}=e^{-F_{1}}\left(\frac{\partial \varphi_{q+1}}{\partial x_{i}}-a_{i q+1}-\sum_{t=2}^{q} a_{i t} \frac{\partial \varphi_{q+1}}{\partial y_{t}}\right)+\sum_{t=2}^{q} a_{i t} \frac{\partial F_{2}^{0}}{\partial y_{t}}-c_{i}^{2}, \quad i=1, \ldots, m$,

где $c_{i}^{2}$ - некоторая функция переменных $x_{1}, \ldots, x_{m}, y_{2}, \ldots, y_{q}$.

Лемма 6.2. Существует функиия $\lambda_{2}=\lambda_{2}\left(x_{1}, \ldots, x_{m}, y_{2}, \ldots, y_{q}\right)$ такая, что функиия $F_{2}=F_{2}^{0}+\lambda_{2}$ удовлетворяет равенству

$$
\frac{\partial F_{2}}{\partial x_{i}}=e^{-F_{1}}\left(\frac{\partial \varphi_{q+1}}{\partial x_{i}}-a_{i q+1}-\sum_{t=2}^{q} a_{i t} \frac{\partial \varphi_{q+1}}{\partial y_{t}}\right)+\sum_{t=2}^{q} a_{i t} \frac{\partial F_{2}}{\partial y_{t}}, \quad i=1, \ldots, m .
$$

Функиия $\lambda_{2}$ находится в явном виде в квадратурах и зависит от въгбора произвольной гладкой функции от $q-1$ переменных.

Лемма 6.2 будет доказана в следуюшем параграфе.

В дальнейшем будем считать, что в формуле (6.7) $\int \frac{\partial \varphi_{q+1}}{\partial y_{1}} e^{-F_{1}} d y_{1}=F_{2}$. Таким образом,

$$
f=\varphi_{q+1}-e^{F_{1}} F_{2}
$$

Проверим, что функция $f$, определенная формулой (6.13), удовлетворяет системе (6.8).

Так как $f$ есть решение уравнения (6.1), то

$$
\frac{\partial f}{\partial y_{1}}=\frac{f-\varphi_{q+1}}{\varphi_{1}}
$$

а потому из (6.13) получаем

$$
\frac{\partial f}{\partial y_{1}}=-\frac{1}{\varphi_{1}} e^{F_{1}} F_{2}
$$


Из (6.13) вытекает равенство

$$
\frac{\partial f}{\partial y_{t}}=\frac{\partial \varphi_{q+1}}{\partial y_{t}}-\frac{\partial F_{1}}{\partial y_{t}} e^{F_{1}} F_{2}-e^{F_{1}} \frac{\partial F_{2}}{\partial y_{t}}, \quad t=2, \ldots, q .
$$

Переходим к вычислению $\frac{\partial f}{\partial x_{i}}, i=1, \ldots, m$.

$$
\begin{aligned}
\frac{\partial f}{\partial x_{i}}= & \frac{\partial \varphi_{q+1}}{\partial x_{i}}-\frac{\partial F_{1}}{\partial x_{i}} e^{F_{1}} F_{2}-e^{F_{1}} \frac{\partial F_{2}}{\partial x_{i}}=\mid \text { см. }(6.10),(6.12) \mid \\
= & \frac{\partial \varphi_{q+1}}{\partial x_{i}}-\left(\frac{a_{i 1}}{\varphi_{1}}+\sum_{t=2}^{q} a_{i t} \frac{\partial F_{1}}{\partial y_{t}}\right) e^{F_{1}} F_{2} \\
& -\frac{\partial \varphi_{q+1}}{\partial x_{i}}+a_{i q+1}+\sum_{t=2}^{q} a_{i t} \frac{\partial \varphi_{q+1}}{\partial y_{t}}-e^{F_{1}} \sum_{t=2}^{q} a_{i t} \frac{\partial F_{2}}{\partial y_{t}} \\
= & a_{i 1}\left(-\frac{1}{\varphi_{1}} e^{F_{1}} F_{2}\right) \\
& +\sum_{t=2}^{q} a_{i t}\left(\frac{\partial \varphi_{q+1}}{\partial y_{t}}-\frac{\partial F_{1}}{\partial y_{t}} e^{F_{1}} F_{2}-e^{F_{1}} \frac{\partial F_{2}}{\partial y_{t}}\right)+a_{i q+1}=\mid \text { cм. (6.14), (6.15)| } \\
= & a_{i 1} \frac{\partial f}{\partial y_{1}}+\sum_{t=2}^{q} a_{i t} \frac{\partial f}{\partial y_{t}}+a_{i q+1}=\sum_{t=1}^{q} a_{i t} \frac{\partial f}{\partial y_{t}}+a_{i q+1},
\end{aligned}
$$

что и доказывает (6.8).

Мы видим, что функция $f$ удовлетворяет системе (4.2), является решением уравнения (4.6) и $\frac{\partial f}{\partial y_{q+1}}=1$. Поэтому $f$ есть однородная функция первой степени однородности. Теорема 6.1 доказана.

\section{§7. Доказательство лемм 6.1 и 6.2}

ДокаЗАТЕльство ЛЕммы 6.1. Прежде всего заметим, что функция $F_{1}=$ $F_{1}^{0}+\lambda_{1}$ удовлетворяет системе (6.10) тогда и только тогда, когда функция $\lambda_{1}$ удовлетворяет системе

$$
\frac{\partial \lambda_{1}}{\partial x_{i}}=\sum_{t=2}^{q} a_{i t} \frac{\partial \lambda_{1}}{\partial y_{t}}+c_{i}^{1}, \quad i=1, \ldots, m .
$$

Нужные нам сведения о системах такого вида изложены в [13; $§ 6]$. В частности, мы можем выписать условия интегрируемости системы (7.1):

$$
\begin{aligned}
\frac{\partial a_{i k}}{\partial x_{j}}-\frac{\partial a_{j k}}{\partial x_{i}} & =\sum_{t=2}^{q}\left(\frac{\partial a_{i k}}{\partial y_{t}} a_{j t}-\frac{\partial a_{j k}}{\partial y_{t}} a_{i t}\right), \quad i, j=1, \ldots, m, k=2, \ldots, q, \\
\frac{\partial c_{i}^{1}}{\partial x_{j}}-\frac{\partial c_{j}^{1}}{\partial x_{i}} & =\sum_{t=2}^{q}\left(\frac{\partial c_{i}^{1}}{\partial y_{t}} a_{j t}-\frac{\partial c_{j}^{1}}{\partial y_{t}} a_{i t}\right), \quad i, j=1, \ldots, m .
\end{aligned}
$$


Из равенств (6.5) следует, что условия (7.2) совпадают с уже доказанными формулами (4.3) при $k=2, \ldots, q$. Остается проверить справедливость условий (7.3).

Из формул (6.9) получаем

$$
c_{i}^{1}=\frac{a_{i 1}}{\varphi_{1}}+\sum_{k=2}^{q} a_{i k} \frac{\partial F_{1}^{0}}{\partial y_{k}}-\frac{\partial F_{1}^{0}}{\partial x_{i}}, \quad i=1, \ldots, m .
$$

Прямое вычисление показьвает, что

$$
\begin{aligned}
& \frac{\partial c_{j}^{1}}{\partial x_{i}}-\frac{\partial c_{i}^{1}}{\partial x_{j}}+\sum_{t=2}^{q}\left(\frac{\partial c_{i}^{1}}{\partial y_{t}} a_{j t}-\frac{\partial c_{j}^{1}}{\partial y_{t}} a_{i t}\right) \\
& =\sum_{k=2}^{q} \frac{\partial F_{1}^{0}}{\partial y_{k}}\left[\frac{\partial a_{j k}}{\partial x_{i}}-\frac{\partial a_{i k}}{\partial x_{j}}+\sum_{t=2}^{q}\left(a_{j t} \frac{\partial a_{i k}}{\partial y_{t}}-a_{i t} \frac{\partial a_{j k}}{\partial y_{t}}\right)\right] \\
& +\frac{\partial}{\partial x_{i}}\left(\frac{a_{j 1}}{\varphi_{1}}\right)-\frac{\partial}{\partial x_{j}}\left(\frac{a_{i 1}}{\varphi_{1}}\right)+\sum_{t=2}^{q}\left(a_{j t} \frac{\partial}{\partial y_{t}}\left(\frac{a_{i 1}}{\varphi_{1}}\right)-a_{i t} \frac{\partial}{\partial y_{t}}\left(\frac{a_{j 1}}{\varphi_{1}}\right)\right) \\
& =|\mathrm{cm} .(4.3),(6.5)| \\
& =\frac{\partial}{\partial x_{i}}\left(\frac{a_{j 1}}{\varphi_{1}}\right)-\frac{\partial}{\partial x_{j}}\left(\frac{a_{i 1}}{\varphi_{1}}\right)+\sum_{t=2}^{q}\left(a_{j t} \frac{\partial}{\partial y_{t}}\left(\frac{a_{i 1}}{\varphi_{1}}\right)-a_{i t} \frac{\partial}{\partial y_{t}}\left(\frac{a_{j 1}}{\varphi_{1}}\right)\right) \\
& =\frac{1}{\varphi_{1}^{2}}\left[\frac{\partial a_{j 1}}{\partial x_{i}} \varphi_{1}-a_{j 1} \frac{\partial \varphi_{1}}{\partial x_{i}}-\frac{\partial a_{i 1}}{\partial x_{j}} \varphi_{1}+a_{i 1} \frac{\partial \varphi_{1}}{\partial x_{j}}\right. \\
& \left.+\sum_{t=2}^{q}\left(a_{j t} \frac{\partial a_{i 1}}{\partial y_{t}} \varphi_{1}-a_{i 1} a_{j t} \frac{\partial \varphi_{1}}{\partial y_{t}}-a_{i t} \frac{\partial a_{j 1}}{\partial y_{t}} \varphi_{1}+a_{j 1} a_{i t} \frac{\partial \varphi_{1}}{\partial y_{t}}\right)\right] \\
& =\frac{1}{\varphi_{1}^{2}}\left[\varphi_{1}\left(\frac{\partial a_{j 1}}{\partial x_{i}}-\frac{\partial a_{i 1}}{\partial x_{j}}+\sum_{t=2}^{q}\left(a_{j t} \frac{\partial a_{i 1}}{\partial y_{t}}-a_{i t} \frac{\partial a_{j 1}}{\partial y_{t}}\right)\right)\right. \\
& \left.+\left(-a_{j 1} \frac{\partial \varphi_{1}}{\partial x_{i}}+a_{i 1} \frac{\partial \varphi_{1}}{\partial x_{j}}+\sum_{t=2}^{q}\left(-a_{i 1} a_{j t} \frac{\partial \varphi_{1}}{\partial y_{t}}+a_{j 1} a_{i t} \frac{\partial \varphi_{1}}{\partial y_{t}}\right)\right)\right] \\
& =\mid \text { см. (4.3) при } k=1 \mid \\
& =\frac{1}{\varphi_{1}^{2}}\left[\varphi_{1}\left(a_{i 1} \frac{\partial a_{j 1}}{\partial y_{1}}-a_{j 1} \frac{\partial a_{i 1}}{\partial y_{1}}\right)\right. \\
& \left.+\left(-a_{j 1} \frac{\partial \varphi_{1}}{\partial x_{i}}+a_{i 1} \frac{\partial \varphi_{1}}{\partial x_{j}}+\sum_{t=2}^{q}\left(-a_{i 1} a_{j t} \frac{\partial \varphi_{1}}{\partial y_{t}}+a_{j 1} a_{i t} \frac{\partial \varphi_{1}}{\partial y_{t}}\right)\right)\right] \\
& =\frac{1}{\varphi_{1}^{2}}\left[a_{i 1}\left(\varphi_{1} \frac{\partial a_{j 1}}{\partial y_{1}}+\frac{\partial \varphi_{1}}{\partial x_{j}}-\sum_{t=2}^{q} a_{j t} \frac{\partial \varphi_{1}}{\partial y_{t}}\right)\right. \\
& \left.+a_{j 1}\left(-\varphi_{1} \frac{\partial a_{i 1}}{\partial y_{1}}-\frac{\partial \varphi_{1}}{\partial x_{i}}+\sum_{t=2}^{q} a_{i t} \frac{\partial \varphi_{1}}{\partial y_{t}}\right)\right] \\
& =|\mathrm{cm} .(6.2)| \\
& =\frac{1}{\varphi_{1}^{2}}\left(a_{i 1} a_{j 1} \frac{\partial \varphi_{1}}{\partial y_{1}}-a_{j 1} a_{i 1} \frac{\partial \varphi_{1}}{\partial y_{1}}\right)=0 \text {. }
\end{aligned}
$$


Итак, условия (7.2) и (7.3) вьполняются. Поэтому к системе (7.1) можно применить метод Майера, который сводит ее решение к решению единственного уравнения.

Заметим, что коэффициенты $a_{i t}, c_{i}^{1}$ и неизвестная функция $\lambda_{1}$ системы $(7.1)$ зависят лишш от переменных $x_{1}, \ldots, x_{m}, y_{2}, \ldots, y_{q}$. При этом в силу следствия $4.1^{\prime}$ коэффициенты $a_{i t}$ можно представить в виде

$$
a_{i t}=a_{i t}^{\prime} y_{t}+a_{i t}^{\prime \prime}, \quad i=1, \ldots, m, \quad t=2, \ldots, q
$$

где функции $a_{i t}^{\prime}, a_{i t}^{\prime \prime}$ не зависят от $y_{t}, y_{t+1}, \ldots, y_{q}$.

Будем искать решение $\lambda_{1}$ системы $(7.1)$, удовлетворяющее начальному условию

$$
\lambda_{1}\left(\xi_{1}, \ldots, \xi_{m}, y_{2}, \ldots, y_{q}\right)=\omega\left(y_{2}, \ldots, y_{q}\right)
$$

где $\xi_{1}, \ldots, \xi_{m}$ - фиксированные значения переменных $x_{1}, \ldots, x_{m}$, a $\omega\left(y_{2}, \ldots, y_{q}\right)-$ произвольная фиксированная гладкая функция аргументов $y_{2}, \ldots, y_{q}$. При некоторых ограничениях на числа $\xi_{1}, \ldots, \xi_{m}$ система (7.1) имеет единственное решение $\lambda_{1}$, удовлетворяюшее начальному условию (7.5). Найти это решение можно методом Майера.

Рассматривается преобразование вида

$$
x_{i}=\xi_{i}+u u_{i}, \quad i=1, \ldots, m
$$

где $u_{1}, \ldots, u_{m}, u$ - новые аргументы.

Неизвестная функция $\lambda_{1}\left(x_{1}, \ldots, x_{m}, y_{2}, \ldots, y_{q}\right)$ превращается в функцию $z=$ $z\left(u_{1}, \ldots, u_{m}, y_{2}, \ldots, y_{q}, u\right)$, которая должна удовлетворять уравнению

$$
\frac{\partial z}{\partial u}-\sum_{k=2}^{q} f_{k} \frac{\partial z}{\partial y_{k}}=h
$$

где

$$
\begin{aligned}
f_{k} & =\sum_{i=1}^{m} u_{i} a_{i k}\left(\xi_{1}+u u_{1}, \ldots, \xi_{m}+u u_{m}, y_{2}, \ldots, y_{q}\right) \\
h & =\sum_{i=1}^{m} u_{i} c_{i}^{1}\left(\xi_{1}+u u_{1}, \ldots, \xi_{m}+u u_{m}, y_{2}, \ldots, y_{q}\right) .
\end{aligned}
$$

Из (7.4) следует, что функцию $f_{k}$ можно записать в виде

$$
f_{k}=f_{k}^{\prime} y_{k}+f_{k}^{\prime \prime}
$$

где $f_{k}^{\prime}, f_{k}^{\prime \prime}$ не зависят от $y_{k}, y_{k+1}, \ldots, y_{q}$. Поэтому уравнение (7.6) принимает следуюший вид:

$$
\frac{\partial z}{\partial u}-\sum_{k=2}^{q}\left(f_{k}^{\prime} y_{k}+f^{\prime \prime}\right) \frac{\partial z}{\partial y_{k}}=h
$$


В уравнении (7.8) $u, y_{2}, \ldots, y_{q}$ выступают в качестве независимых переменных, а $u_{1}, \ldots, u_{m}-$ в качестве параметров.

Из начального условия (7.5) для $\lambda_{1}$ следует, что

$$
z\left(u_{1}, \ldots, u_{m}, y_{2}, \ldots, y_{q}, 0\right)=\omega\left(y_{2}, \ldots, y_{q}\right)
$$

Пусть $z=z\left(u_{1}, \ldots, u_{m}, y_{2}, \ldots, y_{q}, u\right)$ - решение уравнения $(7.8)$ с начальным условием (7.9). Тогда функция

$$
\lambda_{1}\left(x_{1}, \ldots, x_{m}, y_{2}, \ldots, y_{q}\right)=z\left(x_{1}-\xi_{1}, \ldots, x_{m}-\xi_{m}, y_{2}, \ldots, y_{q}, 1\right)
$$

есть решение системы (7.1) с начальным условием (7.5).

Для того чтобы найти решение уравнения (7.8) с начальным условием (7.9), рассмотрим систему

$$
\frac{d u}{1}=-\frac{d y_{2}}{f_{2}^{\prime} y_{2}+f_{2}^{\prime \prime}}=\cdots=-\frac{d y_{q}}{f_{q}^{\prime} y_{q}+f_{q}^{\prime \prime}}=\frac{d z}{h} .
$$

И пусть

$$
\left\{\begin{array}{c}
\psi_{1}\left(z, y_{2}, \ldots, y_{q}, u\right)=C_{1} \\
\ldots \ldots \ldots \ldots \ldots \ldots \ldots \\
\psi_{q}\left(z, y_{2}, \ldots, y_{q}, u\right)=C_{q}
\end{array}\right.
$$

- система независимых интегралов системы (7.11). Положим

$$
\left\{\begin{array}{l}
\bar{\psi}_{1}=\psi_{1}\left(z, y_{2}, \ldots, y_{q}, 0\right) \\
\ldots \ldots \ldots \ldots \ldots \ldots \ldots \\
\bar{\psi}_{q}=\psi_{q}\left(z, y_{2}, \ldots, y_{q}, 0\right)
\end{array}\right.
$$

Можно считать, что (7.13) позволяет выразить $z, y_{2}, \ldots, y_{q}$ через $\bar{\psi}_{1}, \ldots, \bar{\psi}_{q}$ :

$$
\left\{\begin{array}{l}
z=\rho_{1}\left(\bar{\psi}_{1}, \ldots, \bar{\psi}_{q}\right) \\
y_{i}=\rho_{i}\left(\bar{\psi}_{1}, \ldots, \bar{\psi}_{q}\right), i=2, \ldots, q
\end{array}\right.
$$

Рассмотрим функццию

$$
V\left(z, y_{2}, \ldots, y_{q}, u\right) \equiv \rho_{1}\left(\psi_{1}, \ldots, \psi_{q}\right)-\omega\left(\rho_{2}\left(\psi_{1}, \ldots, \psi_{q}\right), \ldots, \rho_{q}\left(\psi_{1}, \ldots, \psi_{q}\right)\right)
$$

Тогда уравнение

$$
V\left(z, y_{2}, \ldots, y_{q}, u\right)=0
$$

определяет $z$ как функцию от $y_{2}, \ldots, y_{q}, u$. Эта функция должна быть решением уравнения (7.8). При $u=0$ функции $\psi_{1}, \ldots, \psi_{q}$ обратятся в $\bar{\psi}_{1}, \ldots, \bar{\psi}_{q}$, функции $\rho_{1}, \rho_{2}, \ldots, \rho_{q}$ дадут соответственно $z, y_{2}, \ldots, y_{q}$, и мы получим, что $z=\omega\left(y_{2}, \ldots, y_{q}\right)$ при $u=0$.

Остается найти систему (7.12) независимых интегралов системы (7.11). 
Если в системе (7.11) принять $u$ за независимую переменную, то система получит следующий вид:

$$
\left\{\begin{array}{l}
\frac{d y_{k}}{d u}+f_{k}^{\prime} y_{k}=-f_{k}^{\prime \prime}, k=2, \ldots, q, \\
\frac{d z}{d u}=h .
\end{array}\right.
$$

Рассмотрим первое уравнение системы (7.16). Согласно $(7.7)$ в нем $f_{2}^{\prime}=f_{2}^{\prime}(u)$, $f_{2}^{\prime \prime}=f_{2}^{\prime \prime}(u)$ и потому оно имеет стандартное решение

$$
y_{2}=e^{-\int f_{2}^{\prime} d u}\left(C_{2}-\int f_{2}^{\prime \prime} e^{\int f_{2}^{\prime} d u} d u\right)
$$

где $C_{2}$ - произвольная постоянная. С помощью (7.17) получается следующий интеграл системы (7.11):

$$
\psi_{2}\left(y_{2}, u\right) \equiv y_{2} e^{\int f_{2}^{\prime} d u}+\int f_{2}^{\prime \prime} e^{\int f_{2}^{\prime} d u} d u=C_{2} .
$$

Рассмотрим второе уравнение системы (7.16). Согласно (7.7) в нем $f_{3}^{\prime}=f_{3}^{\prime}\left(y_{2}, u\right)$, $f_{3}^{\prime \prime}=f_{3}^{\prime \prime}\left(y_{2}, u\right)$. Если воспользоваться формулой $(7.17)$, то $f_{3}^{\prime}, f_{3}^{\prime \prime}$ превратятся в функции $\widetilde{f}_{3}^{\prime}=\widetilde{f}_{3}^{\prime}\left(u, C_{2}\right), \widetilde{f}_{3}^{\prime \prime}=\widetilde{f}_{3}^{\prime \prime}\left(u, C_{2}\right)$, так что

$$
y_{3}=e^{-\int \widetilde{f}_{3}^{\prime} d u}\left(C_{3}-\int \widetilde{f}_{3}^{\prime \prime} e^{\int \widetilde{f}_{3}^{\prime} d u} d u\right),
$$

откуда получаем еще один интеграл системы (7.11):

$$
\left.\psi_{3}\left(y_{2}, y_{3}, u\right) \equiv\left(y_{3} e^{\int \widetilde{f}_{3}^{\prime} d u}+\int \tilde{f}_{3}^{\prime \prime} e^{\int \tilde{f}_{3}^{\prime} d u} d u\right)\right|_{C_{2}=\psi_{2}\left(y_{2}, u\right)}=C_{3} .
$$

В третьем уравнении системы (7.16) $f_{4}^{\prime}=f_{4}^{\prime}\left(y_{2}, y_{3}, u\right), f_{4}^{\prime \prime}=f_{4}^{\prime \prime}\left(y_{2}, y_{3}, u\right)$. Если воспользоваться формулами (7.17) и (7.18), то $f_{4}^{\prime}, f_{4}^{\prime \prime}$ превратятся в функции $\widetilde{f}_{4}^{\prime}=\widetilde{f}_{4}^{\prime}\left(u, C_{2}, C_{3}\right), \widetilde{f}_{4}^{\prime \prime}=\widetilde{f}_{4}^{\prime \prime}\left(u, C_{2}, C_{3}\right)$ и т. д. В результате получим интегралы $\psi_{2}, \ldots, \psi_{q}$ системы $(7.11)$, не зависящие от $z$.

Переходя к последнему уравнению системы (7.16), из функции $h=h\left(y_{2}, \ldots, y_{q}, u\right)$ аналогичным образом получим функции $\widetilde{h}=\widetilde{h}\left(u, C_{2}, \ldots, C_{q}\right)$. Тогда

$$
z=\int \widetilde{h} d u+C_{1}
$$

откуда получаем последний недостающий интеграл системы (7.11):

$$
\psi_{1}\left(z, y_{2}, \ldots, y_{q}, u\right) \equiv z-\left.\int \widetilde{h} d u\right|_{\substack{C_{i}=\psi_{i}, i=2, \ldots, q}}=C_{1}
$$

Очевидно, интегралы $\psi_{1}, \psi_{2}, \ldots, \psi_{q}$ независимы и определены в явном виде в квадратурах. Очевидно также, что найденная система интегралов (7.12) позволяет выразить $z, y_{2}, \ldots, y_{q}$ через $\bar{\psi}_{1}, \ldots, \bar{\psi}_{q}$ в явном виде (см. (7.13) и (7.14)). Наконец, уравнение (7.15) определяет $z$ как функцию от $y_{2}, \ldots, y_{q}$ и снова в явном виде. После этого искомая функция $\lambda_{1}=\lambda_{1}\left(x_{1}, \ldots, x_{m}, y_{2}, \ldots, y_{q}\right)$ определяется формулой (7.10).

Мы видим, что $\lambda_{1}$ определяется в явном виде в квадратурах и зависит от выбора функции $\omega\left(y_{2}, \ldots, y_{q}\right)$. Лемма 6.1 доказана. 
ДокАЗАТЕЛьство ЛЕммы 6.2. Заметим сначала, что функция $F_{2}=F_{2}^{0}+\lambda_{2}$ удовлетворяет системе (6.12) тогда и только тогда, когда функция $\lambda_{2}$ удовлетворяет системе

$$
\frac{\partial \lambda_{2}}{\partial x_{i}}=\sum_{t=2}^{q} a_{i t} \frac{\partial \lambda_{2}}{\partial y_{t}}+c_{i}^{2}, \quad i=1, \ldots, m
$$

Проверка выполнения условий интегрируемости системы (7.19) сводится к проверке справедливости следующих равенств, аналогичных равенствам (7.3) для системы (7.1):

$$
\frac{\partial c_{i}^{2}}{\partial x_{j}}-\frac{\partial c_{j}^{2}}{\partial x_{i}}=\sum_{t=2}^{q}\left(\frac{\partial c_{i}^{2}}{\partial y_{t}} a_{j t}-\frac{\partial c_{j}^{2}}{\partial y_{t}} a_{i t}\right), \quad i, j=1, \ldots, m
$$

Из формул (6.11) получаем:

$c_{i}^{2}=e^{-F_{1}}\left(\frac{\partial \varphi_{q+1}}{\partial x_{i}}-a_{i q+1}-\sum_{k=2}^{q} a_{i k} \frac{\partial \varphi_{q+1}}{\partial y_{k}}\right)+\sum_{k=2}^{q} a_{i k} \frac{\partial F_{2}^{0}}{\partial y_{k}}-\frac{\partial F_{2}^{0}}{\partial x_{i}}, \quad i=1, \ldots, m$

Прямое вычисление дает:

$$
\begin{aligned}
\frac{\partial c_{j}^{2}}{\partial x_{i}} & -\frac{\partial c_{i}^{2}}{\partial x_{j}}+\sum_{t=2}^{q}\left(\frac{\partial c_{i}^{2}}{\partial y_{t}} a_{j t}-\frac{\partial c_{j}^{2}}{\partial y_{t}} a_{i t}\right) \\
= & \sum_{k=2}^{q} \frac{\partial F_{2}^{0}}{\partial y_{k}}\left[\frac{\partial a_{j k}}{\partial x_{i}}-\frac{\partial a_{i k}}{\partial x_{j}}+\sum_{t=2}^{q}\left(a_{j t} \frac{\partial a_{i k}}{\partial y_{t}}-a_{i t} \frac{\partial a_{j k}}{\partial y_{t}}\right)\right] \\
& +e^{-F_{1}}\left[-\frac{\partial F_{1}}{\partial x_{i}}\left(\frac{\partial \varphi_{q+1}}{\partial x_{j}}-a_{j q+1}-\sum_{k=2}^{q} a_{j k} \frac{\partial \varphi_{q+1}}{\partial y_{k}}\right)\right. \\
& -\left(\frac{\partial a_{j q+1}}{\partial x_{i}}+\sum_{k=2}^{q} \frac{\partial a_{j k}}{\partial x_{i}} \frac{\partial \varphi_{q+1}}{\partial y_{k}}\right) \\
& +\frac{\partial F_{1}}{\partial x_{j}}\left(\frac{\partial \varphi_{q+1}}{\partial x_{i}}-a_{i q+1}-\sum_{k=2}^{q} a_{i k} \frac{\partial \varphi_{q+1}}{\partial y_{k}}\right)+\left(\frac{\partial a_{i q+1}}{\partial x_{j}}+\sum_{k=2}^{q} \frac{\partial a_{i k}}{\partial x_{j}} \frac{\partial \varphi_{q+1}}{\partial y_{k}}\right) \\
& -\sum_{t=2}^{q} a_{j t} \frac{\partial F_{1}}{\partial y_{t}}\left(\frac{\partial \varphi_{q+1}}{\partial x_{i}}-a_{i q+1}-\sum_{k=2}^{q} a_{i k} \frac{\partial \varphi_{q+1}}{\partial y_{k}}\right) \\
& -\sum_{t=2}^{q} a_{j t}\left(\frac{\partial a_{i q+1}}{\partial y_{t}}+\sum_{k=2}^{q} \frac{\partial a_{i k}}{\partial y_{t}} \frac{\partial \varphi_{q+1}}{\partial y_{k}}\right) \\
& +\sum_{t=2}^{q} a_{i t} \frac{\partial F_{1}}{\partial y_{t}}\left(\frac{\partial \varphi_{q+1}}{\partial x_{j}}-a_{j}\right),(6.5) \mid \\
& \left.+\sum_{q+1}^{q} a_{i t}\left(\frac{\partial a_{j}}{\partial y_{t}}+\sum_{k=2}^{q} \frac{\partial a_{j k}}{\partial y_{t}} \frac{\partial \varphi_{q+1}}{\partial y_{k}}\right)\right] \\
& \left.\frac{\partial \varphi_{q+1}}{\partial y_{k}}\right)
\end{aligned}
$$




$$
\begin{aligned}
= & e^{-F_{1}}\left[\left(\frac{\partial \varphi_{q+1}}{\partial x_{j}}-a_{j q+1}-\sum_{k=2}^{q} a_{j k} \frac{\partial \varphi_{q+1}}{\partial y_{k}}\right)\left(-\frac{\partial F_{1}}{\partial x_{i}}+\sum_{t=2}^{q} a_{i t} \frac{\partial F_{1}}{\partial y_{t}}\right)\right. \\
& +\left(\frac{\partial \varphi_{q+1}}{\partial x_{i}}-a_{i q+1}-\sum_{k=2}^{q} a_{i k} \frac{\partial \varphi_{q+1}}{\partial y_{k}}\right)\left(\frac{\partial F_{1}}{\partial x_{j}}-\sum_{t=2}^{q} a_{j t} \frac{\partial F_{1}}{\partial y_{t}}\right) \\
& +\left(\frac{\partial a_{i q+1}}{\partial x_{j}}-\frac{\partial a_{j q+1}}{\partial x_{i}}+\sum_{t=2}^{q}\left(a_{i t} \frac{\partial a_{j q+1}}{\partial y_{t}}-a_{j t} \frac{\partial a_{i q+1}}{\partial y_{t}}\right)\right) \\
& \left.+\sum_{k=2}^{q} \frac{\partial \varphi_{q+1}}{\partial y_{k}}\left(\frac{\partial a_{i k}}{\partial x_{j}}-\frac{\partial a_{j k}}{\partial x_{i}}+\sum_{t=2}^{q}\left(a_{i t} \frac{\partial a_{j k}}{\partial y_{t}}-a_{j t} \frac{\partial a_{i k}}{\partial y_{t}}\right)\right)\right] \\
= & |\mathrm{cm} .(6.10),(4.3),(6.5)| \\
= & e^{-F_{1}}\left[-\left(\frac{\partial \varphi_{q+1}}{\partial x_{j}}-a_{j}\right)\right] \\
& +\left(\frac{\partial \varphi_{q+1}}{\partial x_{i}}-\sum_{i q+1}^{q} a_{j k} \frac{\partial \varphi_{q+1}}{\partial y_{k}}\right) \frac{a_{i 1}}{\varphi_{1}} \\
= & \left.\frac{1}{\varphi_{1}} e^{-F_{1}}\left(-a_{i 1} a_{j 1} \frac{\partial \varphi_{q+1}}{\partial y_{1}}+a_{j 1} \frac{\left.\partial a_{i 1} \frac{\partial \varphi_{q+1}}{\partial y_{1}}\right)}{\partial y_{k}}\right) \frac{a_{j 1}}{\varphi_{1}}+\left(a_{j 1} \frac{\partial a_{i q+1}}{\partial y_{1}}-a_{i 1} \frac{\partial a_{j q+1}}{\partial y_{1}}\right)\right] \\
= & e^{-F_{1}}\left[-\frac{a_{i 1}}{\varphi_{1}}\left(\frac{\partial \varphi_{q+1}}{\partial x_{j}}-\sum_{k=2}^{q} a_{j k} \frac{\partial \varphi_{q+1}}{\partial y_{k}}+\frac{\partial a_{j q+1}}{\partial y_{1}} \varphi_{1}-a_{j q+1}\right)\right. \\
& \left.+\frac{a_{j 1}}{\varphi_{1}}\left(\frac{\partial \varphi_{q+1}}{\partial x_{i}}-\sum_{k=2} a_{i k} \frac{\partial \varphi_{q+1}}{\partial y_{k}}+\frac{\partial a_{i q+1}}{\partial y_{1}} \varphi_{1}-a_{i q+1}\right)\right] \\
& \mid 6.4) \mid
\end{aligned}
$$

Итак, условия (7.20) действительно выполняются.

Сравнивая систему (7.19) с уже изученной системой (7.1), приходим к выводу, что если в решении системы (7.1) методом Майера заменить $c_{i}^{1}$ на $c_{i}^{2}$, то получится искомое решение системы (7.19). Лемма 6.2 доказана.

ЗАмечАниЕ 7.1. Завершая рассмотрение случая $\varphi_{1} \neq 0, \varphi_{2}=\cdots=\varphi_{q}=0$, важно отметить для дальнейшего, что при его изучении мы пользовались не теоремой 4.1, а лишш ее следствием $4.1^{\prime}$.

\section{§ 8. Алгебры произвольного индекса. Общий случай}

Нам осталось рассмотреть случай, когда среди функций $\varphi_{1}, \ldots, \varphi_{q}$ отличны от нуля не менее двух функций. Оказывается, что с помощью подходящей замены переменных этот случай сводится к уже разобранному случаю, когда отлична от нуля лишь одна из функций $\varphi_{1}, \ldots, \varphi_{q}$.

Нам понадобятся некоторые дополнительные сведения об однородных функциAx.

ЛЕмма 8.1. Если $f=f\left(x_{1}, \ldots, x_{n}\right)$ - гладкая однородная функция степени 
однородности $k$, то $\frac{\partial f}{\partial x_{i}}, i=1, \ldots, n,-$ однородная функиия степени однородности $k-1$.

ДОКАЗАТЕЛЬСТво. По условию

$$
x_{1} \frac{\partial f}{\partial x_{1}}+\cdots+x_{n} \frac{\partial f}{\partial x_{n}}=k f .
$$

Продифференцируем левую и правую части (8.1) по $x_{i}$. Получим

$$
\frac{\partial f}{\partial x_{i}}+x_{1} \frac{\partial}{\partial x_{i}}\left(\frac{\partial f}{\partial x_{1}}\right)+\cdots+x_{n} \frac{\partial}{\partial x_{i}}\left(\frac{\partial f}{\partial x_{n}}\right)=k \frac{\partial f}{\partial x_{i}}
$$

откуда

$$
x_{1} \frac{\partial}{\partial x_{1}}\left(\frac{\partial f}{\partial x_{i}}\right)+\cdots+x_{n} \frac{\partial}{\partial x_{n}}\left(\frac{\partial f}{\partial x_{i}}\right)=(k-1) \frac{\partial f}{\partial x_{i}} .
$$

Последнее равенство означает, что $\frac{\partial f}{\partial x_{i}}$ - однородная функция степени однородности $k-1$. Лемма 8.1 доказана.

ЛЕмма 8.2. Если $f=f\left(x_{1}, \ldots, x_{n}\right), n \geqslant 2,-$ гладкая однородная функиия степени однородности $k$, то для кажсдого $i=1, \ldots, n$ существует гладкая однородная функиия $F=F\left(x_{1}, \ldots, x_{n}\right)$ степени однородности $k+1$ такая, что $\frac{\partial F}{\partial x_{i}}=f$. Функиия $F$ определяется в явном виде в квадратурах.

ДокаЗАТЕЛЬство. Будем считать, что $i=n$. И пусть $F_{0}=F_{0}\left(x_{1}, \ldots, x_{n}\right)$ - какая-либо гладкая функция, для которой $\frac{\partial F_{0}}{\partial x_{n}}=f$. Если $F_{0}$ неоднородна, то попробуем подобрать такую гладкую функцию $\lambda=\lambda\left(x_{1}, \ldots, x_{n-1}\right)$, что $F=$ $F_{0}+\lambda$.

$F$ будет однородной функцией степени однородности $k+1$ тогда и только тогда, когда

$$
\sum_{i=1}^{n} x_{i} \frac{\partial F}{\partial x_{i}}=(k+1) F
$$

По условию

$$
\sum_{i=1}^{n} x_{i} \frac{\partial f}{\partial x_{i}}=k f
$$

Так как $F_{0}=\int f d x_{n}$, то

$$
\frac{\partial F_{0}}{\partial x_{i}}=\int \frac{\partial f}{\partial x_{i}} d x_{n}, \quad i=1, \ldots, n-1
$$

Рассмотрим левую часть (8.2).

$$
\sum_{i=1}^{n} x_{i} \frac{\partial F}{\partial x_{i}}=\sum_{i=1}^{n} x_{i} \frac{\partial F_{0}}{\partial x_{i}}+\sum_{i=1}^{n-1} x_{i} \frac{\partial \lambda}{\partial x_{i}} .
$$


Из (8.3) и (8.4) выводится, что

$$
\sum_{i=1}^{n} x_{i} \frac{\partial F_{0}}{\partial x_{i}}=(k+1) F_{0}-\delta
$$

где $\delta$ - некоторая гладкая функция переменных $x_{1}, \ldots, x_{n-1}$. Из (8.2), (8.5) и (8.6) получаем, что $\lambda$ является искомой функцией тогда и только тогда, когда она удовлетворяет уравнению

$$
\sum_{i=1}^{n-1} x_{i} \frac{\partial \lambda}{\partial x_{i}}=(k+1) \lambda+\delta .
$$

Уравнение (8.7) есть линейное неоднородное уравнение с частньми производными первого порядка. Изложим кратко, следуя [15], метод решения уравнений такого типа.

Рассматривается уравнение

$$
\varphi_{1} \frac{\partial z}{\partial x_{1}}+\cdots+\varphi_{n} \frac{\partial z}{\partial x_{n}}=\psi
$$

где $z=z\left(x_{1}, \ldots, x_{n}\right) ; \varphi_{1}, \ldots, \varphi_{n}, \psi$ - функции от $x_{1}, \ldots, x_{n}, z$. Уравнение $(8.8)$ может быть приведено к однородному линейному уравнению следующим приемом. Мы будем искать удовлетворяющую уравнению (8.8) функцию $z$ в неявном виде:

$$
v\left(z, x_{1}, \ldots, x_{n}\right)=0
$$

так что искомой функцией будет $v$. При этом $v$ должно удовлетворять соотношению

$$
\varphi_{1} \frac{\partial v}{\partial x_{1}}+\cdots+\varphi_{n} \frac{\partial v}{\partial x_{n}}+\psi \frac{\partial v}{\partial z}=0 .
$$

Если мы потребуем, чтобы $v$ удовлетворяло (8.9) тождественно относительно $x_{1}, \ldots, x_{n}, z$, то (8.9) окажется линейным однородным уравнением с искомой функцией $v$ от $n+1$ независимых переменных $x_{1}, \ldots, x_{n}, z$.

Рассмотрим систему обыкновенных дифференциальных уравнений, соответствующую уравнению (8.9):

$$
\frac{d x_{1}}{\varphi_{1}}=\cdots=\frac{d x_{n}}{\varphi_{n}}=\frac{d z}{\psi} .
$$

Эта система имеет $n$ независимых (локально определенных) первых интегралов

$$
\left\{\begin{array}{c}
\gamma_{1}\left(z, x_{1}, \ldots, x_{n}\right)=C_{1} \\
\ldots \ldots \ldots \ldots \ldots \ldots \ldots \\
\gamma_{n}\left(z, x_{1}, \ldots, x_{n}\right)=C_{n}
\end{array}\right.
$$

Обшее решение уравнения (8.9) имеет вид:

$$
v=\Phi\left(\gamma_{1}, \ldots, \gamma_{n}\right)
$$


где $\Phi$ - произвольная гладкая функция. Уравнение

$$
\Phi\left(\gamma_{1}, \ldots, \gamma_{n}\right)=0
$$

определяет $z$ как функцию от $x_{1} \ldots, x_{n}$, причем эта функция удовлетворяет уравнению (8.8).

Вернемся к уравнению (8.7). Оно приводится к однородному линейному уравнению

$$
\sum_{i=1}^{n-1} x_{i} \frac{\partial v}{\partial x_{i}}+((k+1) \lambda+\delta) \frac{\partial v}{\partial \lambda}=0
$$

где $v=v\left(\lambda, x_{1}, \ldots, x_{n-1}\right)$. Рассмотрим систему обыкновенных дифференциальных уравнений, соответствующую уравнению (8.11):

$$
\frac{d x_{1}}{x_{1}}=\cdots=\frac{d x_{n-1}}{x_{n-1}}=\frac{d \lambda}{(k+1) \lambda+\delta} .
$$

Считая $x_{1}$ независимой переменной, перепишем (8.12) в виде

$$
\left\{\begin{array}{l}
\frac{d x_{2}}{d x_{1}}=\frac{x_{2}}{x_{1}} \\
\cdots \ldots \ldots \ldots \ldots \ldots \\
\frac{d x_{n-1}}{d x_{1}}=\frac{x_{n-1}}{x_{1}}, \\
\frac{d \lambda}{d x_{1}}=\frac{(k+1) \lambda+\delta}{x_{1}}
\end{array}\right.
$$

Из первых уравнений системы (8.13) получаем

$$
x_{2}=C_{2} x_{1}, \ldots, x_{n-1}=C_{n-1} x_{1},
$$

где $C_{2}, \ldots, C_{n-1}-$ произвольные константы. С учетом (8.14) последнее уравнение (8.13) принимает вид

$$
\frac{d \lambda}{d x_{1}}-(k+1) \frac{1}{x_{1}} \lambda=\frac{\widetilde{\delta}}{x_{1}},
$$

где $\widetilde{\delta}=\widetilde{\delta}\left(x_{1}, C_{2}, \ldots, C_{n-1}\right)=\delta\left(x_{1}, C_{2} x_{1}, \ldots, C_{n-1} x_{1}\right)$. Уравнение $(8.15)$ есть частньй случай уравнения

$$
\frac{d y}{d x}+P y=Q
$$

которое имеет первый интеграл

$$
y e^{-\int P d x}-\int Q e^{\int P d x} d x=C .
$$

Поэтому уравнение (8.15) имеет следующий первый интеграл:

$$
\lambda x_{1}^{k+1}-\int \frac{\widetilde{\delta}}{x_{1}^{k+2}} d x_{1}=C_{n} .
$$


Так как $C_{i}=x_{i} / x_{1}, i=2, \ldots, n-1$, то

$$
\lambda x_{1}^{k+1}-\left.\int \frac{\widetilde{\delta}}{x_{1}^{k+2}} d x_{1}\right|_{C_{i}=x_{i} / x_{1}}=C_{n}
$$

есть первый интеграл системы (8.12), зависящий от $\lambda$. Поэтому согласно теории функция

$$
\lambda=\left.\frac{1}{x_{1}^{k+1}} \int \frac{\widetilde{\delta}}{x_{1}^{k+2}} d x_{1}\right|_{C_{i}=x_{i} / x_{1}}
$$

должна удовлетворять уравнению (8.7). Лемма 8.2 доказана.

Вернемся к уравнению (4.6):

$$
\varphi_{1} \frac{\partial f}{\partial y_{1}}+\cdots+\varphi_{q} \frac{\partial f}{\partial y_{q}}=f-\varphi_{q+1}
$$

Для простоты обозначений будем считать в дальнейшем, что функции $\varphi_{1}, \ldots, \varphi_{q}$ отличны от нуля.

Лемма 8.3. Функиии $\varphi_{k}, k=1, \ldots, q$, имеют следующий вид:

$$
\varphi_{k}=\varphi_{k}^{\prime} y_{k}+\varphi_{k}^{\prime \prime}
$$

где $\varphi_{k}^{\prime}$ и $\varphi_{k}^{\prime \prime}$ - однороднъе функиии степени однородности 0 и 1 соответственно, не зависящие от переменных $y_{k}, y_{k+1}, \ldots, y_{q}$.

ДокАЗАТЕльСтво. По определению $\varphi_{k}=\sum_{i=1}^{m} x_{i} a_{i k}+y_{k}$. Согласно следствию $4.1^{\prime} a_{i k}=a_{i k}^{\prime} y_{k}+a_{i k}^{\prime \prime}, i=1, \ldots, m$, где $a_{i k}^{\prime}$ и $a_{i k}^{\prime \prime}$ не зависят от $y_{k}, \ldots, y_{q}$. Поэтому $\varphi_{k}$ можно записать в виде $\varphi_{k}=\varphi_{k}^{\prime} y_{k}+\varphi_{k}^{\prime \prime}$, где $\varphi_{k}^{\prime}=\sum_{i=1}^{m} x_{i} a_{i k}^{\prime}+1$, $\varphi_{k}^{\prime \prime}=\sum_{i=1}^{m} x_{i} a_{i k}^{\prime \prime}$, причем $\varphi_{k}^{\prime}$ и $\varphi_{k}^{\prime \prime}$ не зависят от $y_{k}, \ldots, y_{q}$.

Так как $\varphi_{k}$ - однородная функция степени однородности 1 и $\frac{\partial \varphi_{k}}{\partial y_{k}}=\varphi_{k}^{\prime}$, то согласно лемме $8.1 \varphi_{k}^{\prime}$ - однородная функция степени однородности 0. Отсюда, в свою очередь, следует, что $\varphi_{k}^{\prime \prime}=\varphi_{k}-\varphi_{k}^{\prime} y_{k}$ есть однородная функция степени однородности 1. Лемма 8.3 доказана.

Рассмотрим однородное уравнение

$$
\varphi_{1} \frac{\partial f}{\partial y_{1}}+\cdots+\varphi_{q} \frac{\partial f}{\partial y_{q}}=0
$$

соответствующее уравнению (4.6). Нас интересуют независимые решения уравнения (8.17). Чтобы их найти, рассмотрим систему обыкновенных дифференциальных уравнений

$$
\frac{d y_{1}}{\varphi_{1}}=\cdots=\frac{d y_{q}}{\varphi_{q}}
$$


Если принять $y_{1}$ за независимую переменную, то система (8.18) принимает вид

$$
\frac{d y_{k}}{d y_{1}}=\frac{\varphi_{k}}{\varphi_{1}}, \quad k=2, \ldots, q
$$

Из леммы 8.3 следует, что систему (8.19) можно записать в виде

$$
\frac{d y_{k}}{d y_{1}}+\left(-\frac{\varphi_{k}^{\prime}}{\varphi_{1}}\right) y_{k}=\frac{\varphi_{k}^{\prime \prime}}{\varphi_{1}}, \quad k=2, \ldots, q
$$

где $\varphi_{1}=\varphi_{1}\left(y_{1}\right), \varphi_{k}^{\prime}=\varphi_{k}^{\prime}\left(y_{1}, \ldots, y_{k-1}\right), \varphi_{k}^{\prime \prime}=\varphi_{k}^{\prime \prime}\left(y_{1}, \ldots, y_{k-1}\right)$.

Рассмотрим первое уравнение системы (8.20). Так как $\varphi_{1}=\varphi_{1}\left(y_{1}\right)$, $\varphi_{2}^{\prime}=\varphi_{2}^{\prime}\left(y_{1}\right), \varphi_{2}^{\prime \prime}=\varphi_{2}^{\prime \prime}\left(y_{1}\right)$, то оно имеет стандартное решение

$$
y_{2}=e^{\int \frac{\varphi_{2}^{\prime}}{\varphi_{1}} d y_{1}}\left(C_{2}+\int \frac{\varphi_{2}^{\prime \prime}}{\varphi_{1}} e^{-\int \frac{\varphi_{2}^{\prime}}{\varphi_{1}} d y_{1}} d y_{1}\right)
$$

где $C_{2}$ - произвольная константа, из которого получаем первьй интеграл системы (8.20):

$$
\gamma_{2} \equiv y_{2} e^{-\int \frac{\varphi_{2}^{\prime}}{\varphi_{1}} d y_{1}}-\int \frac{\varphi_{2}^{\prime \prime}}{\varphi_{1}} e^{-\int \frac{\varphi_{2}^{\prime}}{\varphi_{1}} d y_{1}} d y_{1}=C_{2}
$$

Рассмотрим второе уравнение системы (8.20). В нем $\varphi_{1}=\varphi_{1}\left(y_{1}\right), \varphi_{3}^{\prime}=\varphi_{3}^{\prime}\left(y_{1}, y_{2}\right)$, $\varphi_{3}^{\prime \prime}=\varphi_{3}^{\prime \prime}\left(y_{1}, y_{2}\right)$. Если воспользоваться формулой $(8.21)$, то $\varphi_{3}^{\prime}$ и $\varphi_{3}^{\prime \prime}$ превратятся в функции $\widetilde{\varphi}_{3}^{\prime}=\widetilde{\varphi}_{3}^{\prime}\left(y_{1}, C_{2}\right), \widetilde{\varphi}_{3}^{\prime \prime}=\widetilde{\varphi}_{3}^{\prime \prime}\left(y_{1}, C_{2}\right)$, так что

$$
y_{3}=e^{\int \frac{\widetilde{\varphi}_{3}^{\prime}}{\varphi_{1}} d y_{1}}\left(C_{3}+\int \frac{\widetilde{\varphi}_{3}^{\prime \prime}}{\varphi_{1}} e^{-\int \frac{\widetilde{\varphi}_{3}^{\prime}}{\varphi_{1}} d y_{1}} d y_{1}\right),
$$

где $C_{3}$ - произвольная константа, откуда получаем еще один интеграл системы (8.20):

$$
\left.\gamma_{3} \equiv\left(y_{3} e^{-\int \frac{\widetilde{\varphi}_{3}^{\prime}}{\varphi_{1}} d y_{1}}-\int \frac{\widetilde{\varphi}_{3}^{\prime \prime}}{\varphi_{1}} e^{-\int \frac{\widetilde{\varphi}_{3}^{\prime}}{\varphi_{1}} d y_{1}} d y_{1}\right)\right|_{C_{2}=\gamma_{2}\left(y_{1}, y_{2}\right)}=C_{3}
$$

В третьем уравнении системы (8.20) $\varphi_{1}=\varphi_{1}(y), \varphi_{4}^{\prime}=\varphi_{4}^{\prime}\left(y_{1}, y_{2}, y_{3}\right), \varphi_{4}^{\prime \prime}=$ $\varphi_{4}^{\prime \prime}\left(y_{1}, y_{2}, y_{3}\right)$. Если воспользоваться формулами $(8.21)$ и $(8.23)$, то $\varphi_{4}^{\prime}$ и $\varphi_{4}^{\prime \prime}$ превратятся в функции $\widetilde{\varphi}_{4}^{\prime}=\widetilde{\varphi}_{4}^{\prime}\left(y_{1}, C_{2}, C_{3}\right), \widetilde{\varphi}_{4}^{\prime \prime}=\widetilde{\varphi}_{4}^{\prime \prime}\left(y_{1}, C_{2}, C_{3}\right)$ и т. д. В результате получим набор из $q-1$ независимых первых интегралов системы (8.18)

$$
\gamma_{k}\left(x_{1}, \ldots, x_{m}, y_{1}, \ldots, y_{k}\right), \quad k=2, \ldots, q
$$

которые согласно теории должны быть решениями уравнения (8.17).

Во всех рассуждениях, связанных с уравнением (8.17), переменные $x_{1}, \ldots, x_{m}$ рассматривались как параметры. Теперь мы снова будем смотреть на них как на независимые переменные и покажем, что функции $\gamma_{2}, \ldots, \gamma_{q}$ можно считать однородными степени однородности 1. 
Из леммы 8.3 следует, что $\frac{\varphi_{k}^{\prime}}{\varphi_{1}}$ и $\frac{\varphi_{k}^{\prime \prime}}{\varphi_{1}}, k=2, \ldots, q,-$ однородные функции степени однородности -1 и 0 соответственно.

Рассмотрим формулу (8.22), определяющую функцию $\gamma_{2}$. Согласно лемме 8.2 функции

$$
F_{2}^{1}=\int \frac{\varphi_{2}^{\prime}}{\varphi_{1}} d y_{1}, \quad F_{2}^{2}=\int \frac{\varphi_{2}^{\prime \prime}}{\varphi_{1}} e^{-F_{2}^{1}} d y_{1}
$$

можно считать гладкими однородными степени однородности 0 и 1 соответственно. Но тогда и

$$
\gamma_{2}=y_{2} e^{-F_{2}^{1}}-F_{2}^{2}
$$

- гладкая однородная функция степени однородности 1 по переменным $x_{1}, \ldots, x_{m}$, $y_{1}, y_{2}$.

Перейдем к формуле (8.24), определяющей функцию $\gamma_{3}$. Учитывая, что $\widetilde{\varphi}_{3}^{\prime}$ и $\widetilde{\varphi}_{3}^{\prime \prime}$ получаются из $\varphi_{3}^{\prime}$ и $\varphi_{3}^{\prime \prime}$ с помощью формулы (8.21), запишем последнюю формулу в виде

$$
y_{2}=e^{F_{2}^{1}}\left(C_{2}+F_{2}^{2}\right) .
$$

Так как правая часть (8.25) - гладкая однородная функция степени однородности 1 по переменным $x_{1}, \ldots, x_{m}, y_{1}, C_{2}$, то $\frac{\widetilde{\varphi}_{3}^{\prime}}{\varphi_{1}}$ и $\frac{\widetilde{\varphi}_{3}^{\prime \prime}}{\varphi_{1}}$ - гладкие однородные функции степени однородности -1 и 0 по переменным $x_{1}, \ldots, x_{m}, y_{1}, C_{2}$. Но тогда по лемме 8.2 функции

$$
\widetilde{F}_{3}^{1}=\int \frac{\widetilde{\varphi}_{3}^{\prime}}{\varphi_{1}} d y_{1}, \quad \widetilde{F}_{3}^{2}=\int \frac{\widetilde{\varphi}_{3}^{\prime \prime}}{\varphi_{1}} e^{-\widetilde{F}_{3}^{1}} d y_{1}
$$

можно считать гладкими однородными степени однородности 0 и 1 соответственно по переменньп $x_{1}, \ldots, x_{m}, y_{1}, C_{2}$. Так как $\gamma_{2}$ - гладкая однородная функция степени однородности 1 , то и функция

$$
\gamma_{3}=\left.\left(y_{3} e^{-\widetilde{F}_{3}^{1}}-\widetilde{F}_{3}^{2}\right)\right|_{C_{2}=\gamma_{2}}
$$

- гладкая однородная функция степени однородности 1 по переменным $x_{1}, \ldots, x_{m}$, $y_{1}, y_{2}, y_{3}$. Совершенно аналогично рассматриваются функции $\gamma_{4}, \ldots, \gamma_{q}$.

Итак, будем считать далее, что функции $\gamma_{2}, \ldots, \gamma_{q}$ выбраны гладкими однородньпи степени однородности 1.

Рассмотрим новые независимые переменные $x_{1}, \ldots, x_{m}, z_{1}, \ldots, z_{q}, z_{q+1}$ :

$$
\begin{aligned}
& z_{1}=y_{1}, z_{2}=\gamma_{2}\left(x_{1}, \ldots, x_{m}, y_{1}, y_{2}\right), \ldots, z_{q}=\gamma_{q}\left(x_{1}, \ldots, x_{m}, y_{1}, \ldots, y_{q}\right), z_{q+1}=y_{q+1}, \\
& y_{1}=z_{1}, y_{2}=h_{2}\left(x_{1}, \ldots, x_{m}, z_{1}, z_{2}\right), \ldots, y_{q}=h_{q}\left(x_{1}, \ldots, x_{m}, z_{1}, \ldots, z_{q}\right), y_{q+1}=z_{q+1} .
\end{aligned}
$$

Легко видеть, что не только $\gamma_{2}, \ldots, \gamma_{q}$, но и $h_{2}, \ldots, h_{q}$ - однородные функции степени однородности 1 , причем их можно выписать в явном виде.

При переходе к новым переменным функция $f\left(x_{1}, \ldots, x_{m}, y_{1}, \ldots, y_{q+1}\right)$ превращается в функцию $g\left(x_{1}, \ldots, x_{m}, z_{1}, \ldots, z_{q+1}\right)=f\left(x_{1}, \ldots, x_{m}, z_{1}, h_{2}, \ldots, h_{q}, z_{q+1}\right)$. Если $f$ удовлетворяет системе (4.2), то функция $g$ будет решением уже другой системы линейных однородных дифференциальных уравнений в частных производных 
первого порядка, причем эта новая система остается якобиевой. Мы покажем, что она имеет тот же вид, что и система (4.2):

$$
\frac{\partial g}{\partial x_{i}}=\sum_{k=1}^{q+1} \bar{a}_{i k} \frac{\partial g}{\partial z_{k}}, \quad i=1, \ldots, m
$$

a ее коэффициенты $\bar{a}_{i k}$ остаются однородными функциями степени однородности 0 и сохраняют основное свойство коэффициентов $a_{i k}$ :

$$
\bar{a}_{i k}=\bar{a}_{i k}^{\prime} z_{k}+\bar{a}_{i k}^{\prime \prime}, \quad i=1, \ldots, m, \quad k=1, \ldots, q
$$

где $\bar{a}_{i k}^{\prime}$ и $\bar{a}_{i k}^{\prime \prime}$ не зависят от $z_{k}, z_{k+1}, \ldots, z_{q+1}$. В то же время система (8.26) имеет важное преимушество перед исходной системой (4.2). Оказывается,

$$
\bar{\varphi}_{k}=\sum_{i=1}^{m} x_{i} \bar{a}_{i k}+z_{k}=0, \quad k=2, \ldots, q .
$$

Все это означает, что система (8.26) обладает всеми свойствами, которыми мы пользовались, изучая систему (4.2) в случае $\varphi_{1} \neq 0, \varphi_{2}=\cdots=\varphi_{q}=0$. Следовательно, можно найти решение системы (8.26) в виде однородной функции $g=g\left(x_{1}, \ldots, x_{m}, z_{1}, \ldots, z_{q+1}\right)$ степени однородности 1, причем $\frac{\partial g}{\partial z_{q+1}}=1$. Возврашаясь к переменным $x_{1}, \ldots, x_{m}, y_{1}, \ldots, y_{q+1}$, получаем функцию $f=$ $f\left(x_{1}, \ldots, x_{m}, y_{1}, \ldots, y_{q+1}\right)$, удовлетворяющую исходной системе (4.2). Так как $f=g\left(x_{1}, \ldots, x_{m}, y_{1}, \gamma_{2}, \ldots, \gamma_{q}, y_{q+1}\right)$ и $\gamma_{2}, \ldots, \gamma_{q}$ - однородные функции степени однородности 1 , то и $f$ - однородная функция степени однородности 1 . Очевидно также, что $\frac{\partial f}{\partial y_{q+1}}=1$. Полученньй таким образом инвариант $f$ и будет искомой недостающей функцией на $\mathbf{G}^{*}$.

Переходя к реализации намеченного выше, напомним (см. $\S 5)$, что если функция $f=f\left(y_{1}, \ldots, y_{n}\right)$ удовлетворяет уравнению

$$
a_{1} \frac{\partial f}{\partial y_{1}}+\cdots+a_{n} \frac{\partial f}{\partial y_{n}}=0
$$

и рассматривается преобразование переменных

$$
z_{i}=\gamma_{i}\left(y_{1}, \ldots, y_{n}\right), \quad y_{i}=h_{i}\left(z_{1}, \ldots, z_{n}\right), \quad i=1, \ldots, n
$$

то функция $g=g\left(z_{1}, \ldots, z_{n}\right), g=f\left(h_{1}\left(z_{1}, \ldots, z_{n}\right), \ldots, h_{n}\left(z_{1}, \ldots, z_{n}\right)\right)$, удовлетворяет уравнению

$$
\left.D\left(\gamma_{1}\right)\right|_{y_{i}=h_{i}} \frac{\partial g}{\partial z_{1}}+\cdots+\left.D\left(\gamma_{n}\right)\right|_{y_{i}=h_{i}} \frac{\partial g}{\partial z_{n}}=0
$$

где

$$
D=a_{1} \frac{\partial}{\partial y_{1}}+\cdots+a_{n} \frac{\partial}{\partial y_{n}}
$$


Если коэффициенты $a_{i}$ уравнения (8.29) - однородные функции степени однородности 0, а $\gamma_{i}$ и $h_{i}$ - однородные функции степени однородности 1 , то из леммы 8.1 следует, что коэффициенты уравнения (8.30) - однородные функции степени однородности 0 .

В рассматриваемой ситуации (см. (4.2))

$$
D=D_{i}=\frac{\partial}{\partial x_{i}}-a_{i 1} \frac{\partial}{\partial y_{1}}-\ldots-a_{i q+1} \frac{\partial}{\partial y_{q+1}}, \quad i=1, \ldots, m
$$

и

$$
\begin{gathered}
D_{i}\left(x_{j}\right)=\delta_{i j}, \quad i, j=1, \ldots, m \\
D_{i}\left(y_{1}\right)=-a_{i 1}, \quad D_{i}\left(y_{q+1}\right)=-a_{i q+1}, \\
D_{i}\left(\gamma_{k}\right)=\frac{\partial \gamma_{k}}{\partial x_{i}}-a_{i 1} \frac{\partial \gamma_{k}}{\partial y_{1}}-\ldots-a_{i k} \frac{\partial \gamma_{k}}{\partial y_{k}}, \quad k=2, \ldots, q .
\end{gathered}
$$

Таким образом, если $f$ удовлетворяет системе (4.2), то функция $g$ будет решением системы (8.26), где

$$
\begin{aligned}
& \bar{a}_{i 1}=\left.a_{i 1}\right|_{y_{1}=z_{1}}, \quad \bar{a}_{i q+1}=\left.a_{i q+1}\right|_{\begin{array}{c}
y_{1}=z_{1} \\
y_{q+1}=z_{q+1} \\
y_{j}=h_{j}
\end{array}}, \\
& \bar{a}_{i k}=\left.\left(-\frac{\partial \gamma_{k}}{\partial x_{i}}+a_{i 1} \frac{\partial \gamma_{k}}{\partial y_{1}}+\cdots+a_{i k} \frac{\partial \gamma_{k}}{\partial y_{k}}\right)\right|_{\substack{y_{1}=z_{1} \\
y_{j}=h_{j}}}, \quad k=2, \ldots, q
\end{aligned}
$$

Проверим, что коэффициенты $\bar{a}_{i k}$ обладают свойством (8.27).

При $k=1 a_{i 1}=a_{i 1}^{\prime}\left(x_{1}, \ldots, x_{m}\right) y_{1}+a_{i 1}^{\prime \prime}\left(x_{1}, \ldots, x_{m}\right)$, откуда

$$
\bar{a}_{i 1}=a_{i 1}^{\prime}\left(x_{1}, \ldots, x_{m}\right) z_{1}+a_{i 1}^{\prime \prime}\left(x_{1}, \ldots, x_{m}\right)
$$

Пусть теперь $k=2, \ldots, q$. Согласно следствию $4.1^{\prime}$

$$
a_{i k}=a_{i k}^{\prime}\left(x_{1}, \ldots, x_{m}, y_{1}, \ldots, y_{k-1}\right) y_{k}+a_{i k}^{\prime \prime}\left(x_{1}, \ldots, x_{m}, y_{1}, \ldots, y_{k-1}\right)
$$

Функцию $\gamma_{k}$ можно записать похожим образом (см. $\left.(8.22),(8.24)\right)$ :

$$
\gamma_{k}=\gamma_{k}^{\prime}\left(x_{1}, \ldots, x_{m}, y_{1}, \ldots, y_{k-1}\right) y_{k}+\gamma_{k}^{\prime \prime}\left(x_{1}, \ldots, x_{m}, y_{1}, \ldots, y_{k-1}\right)
$$

Легко видеть, что и функция $h_{k}$ имеет аналогичную структуру:

$$
h_{k}=h_{k}^{\prime}\left(x_{1}, \ldots, x_{m}, z_{1}, \ldots, z_{k-1}\right) z_{k}+h_{k}^{\prime \prime}\left(x_{1}, \ldots, x_{m}, z_{1}, \ldots, z_{k-1}\right)
$$

Из $(8.31),(8.32)$ и $(8.33)$ получаем, что $\bar{a}_{i k}$ записывается в виде

$$
\bar{a}_{i k}=\left.\left(\psi^{\prime}\left(x_{1}, \ldots, x_{m}, y_{1}, \ldots, y_{k-1}\right) y_{k}+\psi^{\prime \prime}\left(x_{1}, \ldots, x_{m}, y_{1}, \ldots, y_{k-1}\right)\right)\right|_{\substack{y_{1}=z_{1} \\ y_{j}=h_{j}}}
$$


откуда с учетом (8.34) получаем искомое представление $\bar{a}_{i k}$ :

$$
\bar{a}_{i k}=\bar{a}_{i k}^{\prime}\left(x_{1}, \ldots, x_{m}, z_{1}, \ldots, z_{k-1}\right) z_{k}+\bar{a}_{i k}^{\prime \prime}\left(x_{1}, \ldots, x_{m}, z_{1}, \ldots, z_{k-1}\right)
$$

Нам осталось проверить справедливость равенств (8.28).

$$
\begin{aligned}
\bar{\varphi}_{k} & =\sum_{i=1}^{m} x_{i} \bar{a}_{i k}+z_{k}=\mid \text { cM. }(8.31) \mid \\
& =\left.\sum_{i=1}^{m} x_{i}\left(a_{i 1} \frac{\partial \gamma_{k}}{\partial y_{1}}+\cdots+a_{i k} \frac{\partial \gamma_{k}}{\partial y_{k}}-\frac{\partial \gamma_{k}}{\partial x_{i}}\right)\right|_{\substack{y_{1}=z_{1} \\
y_{j}=h_{j}}}+z_{k} \\
& =\left.\left(\left(\sum_{i=1}^{m} x_{i} a_{i 1}\right) \frac{\partial \gamma_{k}}{\partial y_{1}}+\cdots+\left(\sum_{i=1}^{m} x_{i} a_{i k}\right) \frac{\partial \gamma_{k}}{\partial y_{k}}-\sum_{i=1}^{m} x_{i} \frac{\partial \gamma_{k}}{\partial x_{i}}\right)\right|_{\substack{y_{1}=z_{1} \\
y_{j}=h_{j}}}+z_{k}
\end{aligned}
$$

Так как $\gamma_{k}=\gamma_{k}\left(x_{1}, \ldots, x_{m}, y_{1}, \ldots, y_{k}\right)$ - однородная функция степени однородности 1 , то

$$
-\sum_{i=1}^{m} x_{i} \frac{\partial \gamma_{k}}{\partial x_{i}}=\sum_{j=1}^{k} y_{j} \frac{\partial \gamma_{k}}{\partial y_{j}}-\gamma_{k}
$$

Поэтому

$$
\begin{aligned}
\bar{\varphi}_{k} & =\left.\left(\left(\sum_{i=1}^{m} x_{i} a_{i 1}+y_{1}\right) \frac{\partial \gamma_{k}}{\partial y_{1}}+\cdots+\left(\sum_{i=1}^{m} x_{i} a_{i k}+y_{k}\right) \frac{\partial \gamma_{k}}{\partial y_{k}}-\gamma_{k}\right)\right|_{\substack{y_{1}=z_{1} \\
y_{j}=h_{j}}}+z_{k} \\
& =\left.\left(\varphi_{1} \frac{\partial \gamma_{k}}{\partial y_{1}}+\cdots+\varphi_{k} \frac{\partial \gamma_{k}}{\partial y_{k}}-\gamma_{k}\right)\right|_{\substack{y_{1}=z_{1} \\
y_{j}=h_{j}}}+z_{k} .
\end{aligned}
$$

Так как $\gamma_{k}$ есть решение уравнения $(8.17)$ и $\frac{\partial \gamma_{k}}{\partial y_{k+1}}=\cdots=\frac{\partial \gamma_{k}}{\partial y_{q}}=0$, то

$$
\varphi_{1} \frac{\partial \gamma_{k}}{\partial y_{1}}+\cdots+\varphi_{k} \frac{\partial \gamma_{k}}{\partial y_{k}}=0
$$

Следовательно,

$$
\bar{\varphi}_{k}=-\gamma_{k} \mid \begin{aligned}
& y_{1}=z_{1} \\
& y_{j}=h_{j}
\end{aligned}
$$

Этим завершается изучение разрешимых алгебр произвольного индекса.

Чтобы убедиться в справедливости основной теоремы (см. введение), достаточно заметить теперь, что если функция $f$ на $\mathbf{G}^{*}$ записывается в каком-либо базисе коалгебры $\mathbf{G}^{*}$ в виде однородной функции степени однородности 1 , то и в любом другом базисе $\mathbf{G}^{*} f$ запишется в том же виде. 


\section{§9. Один пример}

При рассмотрении конкретных примеров следует иметь в виду, что вопрос о несводимости данной разрешимой алгебры $\mathbf{G}$ требует специального изучения. Можно, однако, действовать по-иному.

Выберем сначала какой-либо канонический базис $e_{1}, \ldots, e_{n}$ алгебры $\mathbf{G}$. Затем рассмотрим $(n-1)$-мерньй идеал $\mathbf{G}_{0}=L\left(e_{1}, \ldots, e_{n-1}\right)$. Если $\operatorname{dim} \mathscr{O}_{0} \neq \operatorname{dim} \mathscr{O}$, то $\mathbf{G}$ сводима к идеалу $\mathbf{G}_{0}$. Если же $\operatorname{dim} \mathscr{O}_{0}=\operatorname{dim} \mathscr{O}$, то проверяем справедливость утверждения теоремы 4.1. В случае положительного ответа нужный инвариант $f$ строится так же, как если бы алгебра $\mathbf{G}$ была несводимой, после чего можно перейти к рассмотрению идеала $\mathbf{G}_{0}$. Если ответ отрицательный, то алгебра $\mathbf{G}$ сводима к одному из своих $(n-1)$-мерных идеалов, который можно указать в явном виде (см. 55$)$.

Пусть $\mathbf{G}-7$-мерная нильпотентная алгебра Ли с базисом $e_{1}, \ldots, e_{7}$ и таблицей умножения

\begin{tabular}{c|ccccccc} 
& $e_{1}$ & $e_{2}$ & $e_{3}$ & $e_{4}$ & $e_{5}$ & $e_{6}$ & $e_{7}$ \\
\hline$e_{1}$ & 0 & 0 & 0 & 0 & 0 & 0 & 0 \\
$e_{2}$ & 0 & 0 & 0 & 0 & 0 & $e_{1}$ & $e_{1}$ \\
$e_{3}$ & 0 & 0 & 0 & 0 & $-e_{1}$ & 0 & $e_{2}$ \\
$e_{4}$ & 0 & 0 & 0 & 0 & $e_{1}$ & $e_{2}$ & 0 \\
$e_{5}$ & 0 & 0 & $e_{1}$ & $-e_{1}$ & 0 & $e_{3}$ & $e_{4}$ \\
$e_{6}$ & 0 & $-e_{1}$ & 0 & $-e_{2}$ & $-e_{3}$ & 0 & $e_{5}$ \\
$e_{7}$ & 0 & $-e_{1}$ & $-e_{2}$ & 0 & $-e_{4}$ & $-e_{5}$ & 0
\end{tabular}

Требуется найти полный инволютивный набор функций на $\mathbf{G}^{*}$.

Ясно, что $e_{1}, \ldots, e_{7}-$ канонический базис $\mathbf{G}$ и $[\mathbf{G}, \mathbf{G}]=L\left(e_{1}, \ldots, e_{5}\right)$. Вьпишем матрицу $A$ системы для определения инвариантов на $\mathbf{G}^{*}$, соответствующей этому базису:

$$
A=\left[\begin{array}{ccccccc}
0 & 0 & 0 & 0 & 0 & 0 & 0 \\
0 & 0 & 0 & 0 & 0 & x_{1} & x_{1} \\
0 & 0 & 0 & 0 & -x_{1} & 0 & x_{2} \\
0 & 0 & 0 & 0 & x_{1} & x_{2} & 0 \\
0 & 0 & x_{1} & -x_{1} & 0 & x_{3} & x_{4} \\
0 & -x_{1} & 0 & -x_{2} & -x_{3} & 0 & x_{5} \\
0 & -x_{1} & -x_{2} & 0 & -x_{4} & -x_{5} & 0
\end{array}\right]
$$

Непосредственно проверяется, что $\operatorname{rang} A=4$. Это означает,что если $\mathscr{O}-$ орбита общего положения из $\mathbf{G}^{*}$, то $\operatorname{dim} \mathscr{O}=4, \operatorname{codim} \mathscr{O}=3$ и, следовательно, ind $\mathbf{G}=3$. Таким образом, полный инволютивньй набор на $\mathbf{G}^{*}$ должен состоять из пяти функций: $r=\frac{1}{2}(7+3)=5$.

Рассмотрим $\mathbf{G}_{0}=L\left(e_{1}, \ldots, e_{6}\right)-6$-мерный идеал алгебры $\mathbf{G}$, и пусть $\mathscr{O}_{0}-$ opбита общего положения из $\mathbf{G}_{0}^{*}$. Если в матрице $A$ зачеркнуть последнюю строку и последний столбец, то получится матрица $A_{0}$ системы для определения инвариантов на $\mathbf{G}_{0}^{*}$, соответствуюшей базису $e_{1}, \ldots, e_{6}$. Очевидно, $\operatorname{rang} A_{0}=4$, так что 
$\operatorname{rang} A_{0}=\operatorname{rang} A$ и потому $\operatorname{dim} \mathscr{O}_{0}=\operatorname{dim} \mathscr{O}$. Как нам известно (см. $\left.\S 1\right)$, в этом случае к полному инволютивному набору на $\mathbf{G}_{0}^{*}$ необходимо добавить еще одну функцию $f$. Мы увидим ниже, что в качестве такой функции можно взять подходящий инвариант на $\mathbf{G}^{*}$.

Ясно, что полный инволютивный набор на $\mathbf{G}_{0}^{*}$ должен состоять из четырех функций. Так как $L\left(e_{1}, \ldots, e_{4}\right)$ - абелева подалгебра $\mathbf{G}_{0}$, то из леммы 1.1 следует, что в качестве таких функций можно взять координатные функции $x_{1}, x_{2}, x_{3}, x_{4}$. Остается определить инвариант $f$.

Система для определения инвариантов на $\mathbf{G}^{*}$ содержит четыре независимых уравнения:

$$
\left\{\begin{array}{l}
x_{1} \frac{\partial f}{\partial x_{6}}+x_{1} \frac{\partial f}{\partial x_{7}}=0 \\
-x_{1} \frac{\partial f}{\partial x_{5}}+x_{2} \frac{\partial f}{\partial x_{7}}=0 \\
x_{1} \frac{\partial f}{\partial x_{3}}-x_{1} \frac{\partial f}{\partial x_{4}}+x_{3} \frac{\partial f}{\partial x_{6}}+x_{4} \frac{\partial f}{\partial x_{7}}=0, \\
-x_{1} \frac{\partial f}{\partial x_{2}}-x_{2} \frac{\partial f}{\partial x_{4}}-x_{3} \frac{\partial f}{\partial x_{5}}+x_{5} \frac{\partial f}{\partial x_{7}}=0 .
\end{array}\right.
$$

В соответствии с общей теорией (см. §4) перепишем ее в виде

$$
\left\{\begin{array}{l}
\frac{\partial f}{\partial x_{2}}=0 \cdot \frac{\partial f}{\partial x_{1}}-\frac{x_{2}}{x_{1}} \frac{\partial f}{\partial x_{4}}+\frac{x_{1} x_{5}-x_{2} x_{3}}{x_{1}^{2}} \frac{\partial f}{\partial x_{7}} \\
\frac{\partial f}{\partial x_{3}}=0 \cdot \frac{\partial f}{\partial x_{1}}+\frac{\partial f}{\partial x_{4}}+\frac{x_{3}-x_{4}}{x_{1}} \frac{\partial f}{\partial x_{7}} \\
\frac{\partial f}{\partial x_{5}}=0 \cdot \frac{\partial f}{\partial x_{1}}+0 \cdot \frac{\partial f}{\partial x_{4}}+\frac{x_{2}}{x_{1}} \frac{\partial f}{\partial x_{7}} \\
\frac{\partial f}{\partial x_{6}}=0 \cdot \frac{\partial f}{\partial x_{1}}+0 \cdot \frac{\partial f}{\partial x_{4}}-\frac{\partial f}{\partial x_{7}}
\end{array}\right.
$$

а затем изменим обозначения: переменные $x_{1}, x_{4}, x_{7}$ будем обозначать через $y_{1}, y_{2}, y_{3}$, а переменные $x_{2}, x_{3}, x_{5}, x_{6}$ - через $x_{1}, x_{2}, x_{3}, x_{4}$ соответственно. В результате система (9.1) примет следующий вид:

$$
\left\{\begin{array}{l}
\frac{\partial f}{\partial x_{1}}=0 \cdot \frac{\partial f}{\partial y_{1}}-\frac{x_{1}}{y_{1}} \frac{\partial f}{\partial y_{2}}+\frac{y_{1} x_{3}-x_{1} x_{2}}{y_{1}^{2}} \frac{\partial f}{\partial y_{3}} \\
\frac{\partial f}{\partial x_{2}}=0 \cdot \frac{\partial f}{\partial y_{1}}+\frac{\partial f}{\partial y_{2}}+\frac{x_{2}-y_{2}}{y_{1}} \frac{\partial f}{\partial y_{3}} \\
\frac{\partial f}{\partial x_{3}}=0 \cdot \frac{\partial f}{\partial y_{1}}+0 \cdot \frac{\partial f}{\partial y_{2}}+\frac{x_{1}}{y_{1}} \frac{\partial f}{\partial y_{3}} \\
\frac{\partial f}{\partial x_{4}}=0 \cdot \frac{\partial f}{\partial y_{1}}+0 \cdot \frac{\partial f}{\partial y_{2}}-\frac{\partial f}{\partial y_{3}}
\end{array}\right.
$$

где $f=f\left(x_{1}, x_{2}, x_{3}, x_{4}, y_{1}, y_{2}, y_{3}\right)$ - неизвестная функция. Так как коэффициенты системы (9.2) имеют вид, указанный в следствии $4.1^{\prime}$, то искомый инвариант $f$ можно найти с помощью общего алгоритма. 
В рассматриваемом случае (см. $§ 4)$

$$
\begin{gathered}
\varphi_{1}=y_{1}, \quad \varphi_{2}=\frac{x_{2} y_{1}-x_{1}^{2}}{y_{1}}+y_{2}, \\
\varphi_{3}=\frac{2 x_{1} x_{3} y_{1}+x_{2}^{2} y_{1}-x_{2} y_{1} y_{2}-x_{1}^{2} x_{2}}{y_{1}^{2}}-x_{4}+y_{3} .
\end{gathered}
$$

При этом вспомогательное уравнение (4.6) принимает вид

$$
\varphi_{1} \frac{\partial f}{\partial y_{1}}+\varphi_{2} \frac{\partial f}{\partial y_{2}}=f-\varphi_{3}
$$

и $\varphi_{1} \neq 0, \varphi_{2} \neq 0$ (см. $\left.\S 8\right)$.

Выпишем однородное уравнение, соответствующее уравнению (9.3):

$$
y_{1} \frac{\partial f}{\partial y_{1}}+\left(y_{2}+\frac{x_{2} y_{1}-x_{1}^{2}}{y_{1}}\right) \frac{\partial f}{\partial y_{2}}=0
$$

Чтобы найти какое-либо решение (9.4), рассмотрим обыкновенное дифференциальное уравнение

$$
\frac{d y_{1}}{y_{1}}=\frac{d y_{2}}{y_{2}+\frac{x_{2} y_{1}-x_{1}^{2}}{y_{1}}},
$$

или

$$
\frac{d y_{2}}{d y_{1}}+\left(-\frac{1}{y_{1}}\right) y_{2}=\frac{x_{2} y_{1}-x_{1}^{2}}{y_{1}^{2}}
$$

Уравнение (9.5) имеет стандартное решение:

$$
y_{2}=e^{\int \frac{1}{y_{1}} d y_{1}}\left(C+\int \frac{x_{2} y_{1}-x_{1}^{2}}{y_{1}^{2}} e^{-\int \frac{1}{y_{1}} d y_{1}} d y_{1}\right) .
$$

Положим

$$
F_{1}=\int \frac{1}{y_{1}} d y_{1}, \quad F_{2}=\int \frac{x_{2} y_{1}-x_{1}^{2}}{y_{1}^{2}} e^{-F_{1}} d y_{1} .
$$

Из (9.6) получаем первый интеграл уравнения (9.5):

$$
\gamma \equiv y_{2} e^{-F_{1}}-F_{2}=C
$$

Следовательно, функция $\gamma$ должна быть решением уравнения (9.4).

Подберем $F_{1}$ и $F_{2}$ так, чтобы $\gamma$ была однородной функцией степени однородности 1. Для этого достаточно, чтобы $F_{1}$ и $F_{2}$ были однородньми функциями степени однородности 0 и 1 соответственно.

Выберем какое-либо гладкое значение $F_{1}^{0}$ первого интеграла, например $F_{1}^{0}=$ $\ln y_{1}$, считая $y_{1}>0$. Так как эта функция не является однородной, то будем искать $F_{1}$ в виде $F_{1}=F_{1}^{0}+\lambda_{1}$, где $\lambda_{1}=\lambda_{1}\left(x_{1}, x_{2}, x_{3}, x_{4}, y_{2}\right)$ (см. лемму 8.2). 
$\lambda_{1}$ является искомой функцией тогда и только тогда, когда она удовлетворяет уравнению (8.7) при $k=-1$ :

$$
\sum_{i=1}^{4} x_{i} \frac{\partial \lambda_{1}}{\partial x_{i}}+y_{2} \frac{\partial \lambda_{1}}{\partial y_{2}}=\delta
$$

где функция $\delta=\delta\left(x_{1}, x_{2}, x_{3}, x_{4}, y_{2}\right)$ определяется из равенства (8.6) при $k=-1$ :

$$
\delta=-\sum_{i=1}^{4} x_{i} \frac{\partial F_{1}^{0}}{\partial x_{i}}-\sum_{i=1}^{2} y_{i} \frac{\partial F_{1}^{0}}{\partial y_{i}}
$$

Таким образом, $\delta=-1$ и

$$
\sum_{i=1}^{4} x_{i} \frac{\partial \lambda_{1}}{\partial x_{i}}+y_{2} \frac{\partial \lambda_{1}}{\partial y_{2}}=-1
$$

Последнее уравнение решается без труда. Например, в качестве $\lambda_{1}$ можно взять функцию $\lambda_{1}=-\ln x_{1}$, считая $x_{1}>0$. В результате

$$
F_{1}=\ln y_{1}-\ln x_{1}=\ln \frac{y_{1}}{x_{1}} \text {. }
$$

Найдем теперь какое-нибудь гладкое значение $F_{2}^{0}$ второго интеграла, учитывая, что $e^{-F_{1}}=x_{1} / y_{1}$. Пусть, например,

$$
F_{2}^{0}=\int \frac{x_{1} x_{2} y_{1}-x_{1}^{3}}{y_{1}^{3}} d y_{1}=\frac{x_{1}^{3}-2 x_{1} x_{2} y_{1}}{2 y_{1}^{2}}
$$

Так как $F_{2}^{0}$ - однородная функция степени однородности 1 , то можно считать, что $F_{2}=F_{2}^{0}$.

В результате получаем следующее решение уравнения (9.4):

$$
\gamma=y_{2} \frac{x_{1}}{y_{1}}+\frac{2 x_{1} x_{2} y_{1}-x_{1}^{3}}{2 y_{1}^{2}}
$$

Сделаем теперь замену переменных: вместо $x_{1}, x_{2}, x_{3}, x_{4}, y_{1}, y_{2}, y_{3}$ рассмотрим новые независимые переменные $x_{1}, x_{2}, x_{3}, x_{4}, z_{1}, z_{2}, z_{3}$, где

$$
z_{1}=y_{1}, \quad z_{2}=\gamma\left(x_{1}, x_{2}, y_{1}, y_{2}\right), \quad z_{3}=y_{3}
$$

При этом

$$
y_{1}=z_{1}, \quad y_{2}=h\left(x_{1}, x_{2}, z_{1}, z_{2}\right), \quad y_{3}=z_{3}
$$

где

$$
h=z_{2} \frac{z_{1}}{x_{1}}+\frac{x_{1}^{2}-2 x_{2} z_{1}}{2 z_{1}} .
$$


Пусть функция $f=f\left(x_{1}, x_{2}, x_{3}, x_{4}, y_{1}, y_{2}, y_{3}\right)$ удовлетворяет системе (9.2). При переходе к новым переменным $f$ преврашается в функцию $g\left(x_{1}, x_{2}, x_{3}, x_{4}, z_{1}, z_{2}, z_{3}\right)$, которая удовлетворяет уже другой системе дифференциальных уравнений. Мы можем найти эту новую систему в соответствии с общей теорией (см. 8 8):

$$
\left\{\begin{aligned}
\frac{\partial g}{\partial x_{1}} & =-\frac{z_{2}}{x_{1}} \frac{\partial g}{\partial z_{2}}+\frac{x_{3} z_{1}-x_{1} x_{2}}{z_{1}^{2}} \frac{\partial g}{\partial z_{3}} \\
\frac{\partial g}{\partial x_{2}} & =\frac{4 x_{1} x_{2} z_{1}-2 z_{1}^{2} z_{2}-x_{1}^{3}}{2 x_{1} z_{1}^{2}} \frac{\partial g}{\partial z_{3}} \\
\frac{\partial g}{\partial x_{3}} & =\frac{x_{1}}{z_{1}} \frac{\partial g}{\partial z_{3}} \\
\frac{\partial g}{\partial x_{4}} & =-\frac{\partial g}{\partial z_{3}}
\end{aligned}\right.
$$

Для системы (9.7)

$$
\bar{\varphi}_{1}=z_{1}, \quad \bar{\varphi}_{2}=0, \quad \bar{\varphi}_{3}=\frac{4 x_{1}^{2} x_{3} z_{1}-3 x_{1}^{3} x_{2}+4 x_{1} x_{2}^{2} z_{1}-2 x_{2} z_{1}^{2} z_{2}}{2 x_{1} z_{1}^{2}}-x_{4}+z_{3}
$$

Таким образом, замена переменных свела случай $\varphi_{1} \neq 0, \varphi_{2} \neq 0$ к случаю $\varphi_{1} \neq 0$, $\varphi_{2}=0$.

Найдем какое-либо решение системы (9.7) в виде однородной функции $g$ степени однородности 1 , удовлетворяюшей условию $\frac{\partial g}{\partial z_{3}}=1$ (см. $\left.\S 6\right)$.

Согласно теореме 6.1 функцию $g$ можно найти в виде

$$
g=\bar{\varphi}_{3}-e^{F_{1}} \cdot F_{2}
$$

где

$$
F_{1}=\int \frac{1}{\bar{\varphi}_{1}} d z_{1}, \quad F_{2}=\int \frac{\partial \bar{\varphi}_{3}}{\partial z_{1}} e^{-F_{1}} d z_{1}
$$

причем функции $F_{1}$ и $F_{2}$ должны удовлетворять следующим дополнительным условиям:

$$
\begin{aligned}
& \frac{\partial F_{1}}{\partial x_{i}}=\frac{\bar{a}_{i 1}}{\bar{\varphi}_{1}}+\bar{a}_{i 2} \frac{\partial F_{1}}{\partial z_{2}}, \quad i=1,2,3,4 \\
& \frac{\partial F_{2}}{\partial x_{i}}=e^{-F_{1}}\left(\frac{\partial \bar{\varphi}_{3}}{\partial x_{i}}-\bar{a}_{i 3}-\bar{a}_{i 2} \frac{\partial \bar{\varphi}_{3}}{\partial z_{2}}\right)+\bar{a}_{i 2} \frac{\partial F_{2}}{\partial z_{2}}, \quad i=1,2,3,4
\end{aligned}
$$

Так как $\bar{\varphi}_{1}=z_{1}$, то в качестве $F_{1}$ естественно взять функцию $F_{1}=\ln z_{1}$. Поскольку $\bar{a}_{i 1}=0, i=1,2,3,4$, и $\frac{\partial F_{1}}{\partial z_{2}}=0$, то $F_{1}$ удовлетворяет условиям (9.9).

Перейдем к определению $F_{2}$. Так как

$$
e^{-F_{1}}=\frac{1}{z_{1}}, \quad \frac{\partial \bar{\varphi}_{3}}{\partial z_{1}}=-2\left(x_{1} x_{3}+x_{2}^{2}\right) \frac{1}{z_{1}^{2}}+3 x_{1}^{2} x_{2} \frac{1}{z_{1}^{3}}
$$


то в качестве $F_{2}$ естественно взять функцию $F_{2}=\left(x_{1} x_{3}+x_{2}^{2}\right) / z_{1}^{2}+x_{1}^{2} x_{2} / z_{1}^{3}$. Непосредственно проверяется, что эта функция удовлетворяет условиям (9.10).

Мы можем теперь выписать функцию $g$ в явном виде:

$$
g=\frac{2 x_{1} x_{3} z_{1}+2 x_{2}^{2} z_{1}-x_{1}^{2} x_{2}}{2 z_{1}^{2}}-\frac{x_{2} z_{2}}{x_{1}}-x_{4}+z_{3} .
$$

Искомое решение $f$ системы (9.2) получается из функции $g$ при переходе к переменнымм $x_{1}, x_{2}, x_{3}, x_{4}, y_{1}, y_{2}, y_{3}$ :

$$
f=\frac{x_{1} x_{3}-x_{2} y_{2}}{y_{1}}-x_{4}+y_{3} .
$$

Мы видим, что $f$ - однородная функция степени однородности 1 , причем $\frac{\partial f}{\partial y_{3}}=1$. Непосредственно проверяется, что $f$ действительно удовлетворяет системе (9.2) без каких-либо ограничений на независимые переменные (кроме $y_{1} \neq 0$ ).

Возврашаясь к первоначальным обозначениям независимых переменных, окончательно получаем, что система функций

$$
x_{1}, x_{2}, x_{3}, x_{4}, \frac{x_{2} x_{5}-x_{3} x_{4}}{x_{1}}-x_{6}+x_{7}
$$

образует полный инволютивный набор на коалгебре $\mathbf{G}^{*}$, состоящий из однородных функций степени однородности 1.

В заключение отметим, что с помощью набора (9.11) можно получить полньй инволютивный набор на $\mathbf{G}^{*}$, состоящий из полиномов. Для этого достаточно заменить в (9.11) последнюю функцию $f$ функцией $x_{1} f$, которая тоже будет инвариантом, поскольку инвариантами являются функции $x_{1}$ и $f$.

\section{Список литературы}

1. Арнольд В. И. Математические методы классической механики. М.: Наука, 1974.

2. Мищенко A.C., Фоменко A. Т. Обобщенный метод Лиувилля интегрирования гамильтоновых систем // Функц. анализ и его прилож. 1978. Т. 12. № 2. С. 46-56.

3. Фоменко A. T. Дифференциальная геометрия и топология. Дополнительные главы. М.: Изд-во МГУ, 1983.

4. Фоменко А. Т. Симплектическая геометрия. Методы и приложения. М.: Изд-во МГУ, 1988.

5. Трофимов В. В., Фоменко А.Т. Алгебра и геометрия интегрируемых гамильтоновых диффференциальных уравнений. М.: Факториал, 1995.

6. Vergne $M$. La structure de Poisson sur l'algébre symmétrique d'une algébre de Lie nilpotente // Bull. Soc. Math. France. 1972. V. 100. № 3. P. 301-335.

7. Архангельский $A$. A. Вполне интегрируемые гамильтоновы системы на группе треугольных матриц // Матем. сб. 1979. Т. 108. №1. С. 134-142.

8. Трофимов В. В. Уравнения Эйлера на борелевских подалгебрах полупростых алгебр Ли // Изв. АН СССР. Сер. матем. 1979. Т. 43. № 3. С. 714-732.

9. Трофимов В. В. Уравнения Эйлера на конечномерных разрешимых группах Ли // Изв. АН СССР. Сер. матем. 1980. Т. 44. № 5. С. 1191-1199.

10. Ле Нгок Тьеуен. Коммутативные наборы функций на орбитах общего положения конечномерных алгебр Ли // УМН. 1983. Т. 38. №1. С. 179-180. 
11. Милованов М. В. О гипотезе Мищенко-Фоменко и интегрируемости разрешимых алгебр Ли // Докл. НАН Беларуси. 1998. Т. 42. № 1. С. 45-49.

12. Смирнов В. И. Курс высшей математики. Т. IV, часть 2. М.: Мир, 1989.

13. Камке Э. Справочник по дифференциальньм уравнениям в частных производных первого порядка. М.: Наука, 1966.

14. Гельфанд И. М. Лекции по линейной алгебре. М.: Наука, 1971.

15. Степанов В. В. Курс диффференциальных уравнений. М.: Физматгиз, 1958.

Белорусский государственный

педагогический университет им. М. Танка
Поступила в редакцию

05.06 .1998 